\title{
Traveling Wave Solutions in a Reaction-Diffusion Epidemic Model
}

\author{
Sheng Wang, ${ }^{1}$ Wenbin Liu, ${ }^{2}$ Zhengguang Guo, ${ }^{1}$ and Weiming Wang ${ }^{1}$ \\ ${ }^{1}$ College of Mathematics and Information Science, Wenzhou University, \\ Wenzhou 325035, China \\ ${ }^{2}$ College of Physics and Electronic Information Engineering, Wenzhou University, \\ Wenzhou 325035, China \\ Correspondence should be addressed to Weiming Wang; weimingwang2003@163.com
}

Received 4 February 2013; Revised 17 March 2013; Accepted 17 March 2013

Academic Editor: Anke Meyer-Baese

Copyright (C) 2013 Sheng Wang et al. This is an open access article distributed under the Creative Commons Attribution License, which permits unrestricted use, distribution, and reproduction in any medium, provided the original work is properly cited.

We investigate the traveling wave solutions in a reaction-diffusion epidemic model. The existence of the wave solutions is derived through monotone iteration of a pair of classical upper and lower solutions. The traveling wave solutions are shown to be unique and strictly monotonic. Furthermore, we determine the critical minimal wave speed.

\section{Introduction}

Recently, great attention has been paid to the study of the traveling wave solutions in reaction-diffusion models [1-17]. In the sense of epidemiology, the traveling wave solutions describe the transition from a disease-free equilibrium to an endemic equilibrium; the existence and nonexistence of nontrivial traveling wave solutions indicate whether or not the disease can spread [11]. The results contribute to predicting the developing tendency of infectious diseases, to determining the key factors of the spread of infectious disease, and to seeking the optimum strategies of preventing and controlling the spread of the infectious diseases [18-21].

Some methods have been used to derive the existence of traveling wave solutions in reaction-diffusion models, and the monotone iteration method has been proved to be an effective one. Such a method reduces the existence of traveling wave solutions to that of an ordered pair of upper-lower solutions $[6,7,9,10,14,15]$.

In [22], Berezovsky and coworkers introduced a simple epidemic model through the incorporation of variable population, disease-induced mortality, and emigration into the classical model of Kermack and McKendrick [23]. The total population $(N)$ is divided into two groups of susceptible $(S)$ and infectious (I); that is to say, $N=S+I$. The model describing the relations between the state variables is

$$
\begin{gathered}
\frac{d S}{d t}=r N\left(1-\frac{N}{K}\right)-\beta \frac{S I}{N}-(\mu+m) S, \\
\frac{d I}{d t}=\beta \frac{S I}{N}-(\mu+d) I,
\end{gathered}
$$

where the reproduction of susceptible follows a logistic equation with the intrinsic growth rate $r$ and the carrying capacity $K, \beta$ denotes the contact transmission rate (the infection rate constant), $\mu$ is the natural mortality; $d$ denotes the diseaseinduced mortality, and $m$ is the per-capita emigration rate of uninfected.

For model (1), the epidemic threshold, the so-called basic reproduction number $R_{0}$, is then computed as $R_{0}=\beta /(\mu+$ $d$ ). The disease will successfully invade when $R_{0}>1$ but will die out if $R_{0}<1 . R_{0}=1$ is usually a threshold whether the disease goes to extinction or goes to an endemic. Large values of $R_{0}$ may indicate the possibility of a major epidemic [19]. In addition, the basic demographic reproductive number $R_{d}$ is given by $R_{d}=r /(\mu+m)$. It can be shown that if $R_{d}>1$ the population grows, while $R_{d} \leq 1$ implies that the population does not survive [22]. 
For simplicity, rescaling model (1) by letting $S \rightarrow S / K$, $I \rightarrow I / K$, and $t \rightarrow t /(\mu+d)$ leads to the following model:

$$
\begin{gathered}
\frac{d S}{d t}=v R_{d}(S+I)[1-(S+I)]-R_{0} \frac{S I}{S+I}-v S, \\
\frac{d I}{d t}=R_{0} \frac{S I}{S+I}-I,
\end{gathered}
$$

where $\nu=(\mu+m) /(\mu+d)$ is defined by the ratio of the average life span of susceptibles to that of infectious.

For details, we refer the reader to [20, 22].

In this paper, we are interested in the existence of traveling wave solutions in the following reaction-diffusion epidemic model [20]:

$$
\begin{gathered}
\frac{\partial S}{\partial t}=\nu R_{d}(S+I)[1-(S+I)]-R_{0} \frac{S I}{S+I}-\nu S+d \frac{\partial^{2} S}{\partial x^{2}} \\
\frac{\partial I}{\partial t}=R_{0} \frac{S I}{S+I}-I+d \frac{\partial^{2} I}{\partial x^{2}} \\
S(x, 0)=S_{0}(x), \quad I(x, 0)=I_{0}(x)
\end{gathered}
$$

where $v, R_{0}, R_{d}$ are all positive constants, $d$ is the diffusion coefficient, and $(x, t) \in R \times R^{+}$.

We are looking for the traveling wave solutions of model (3) with the following form:

$$
S(x, t)=S(\xi), \quad I(x, t)=I(\xi), \quad \xi=x+c t,
$$

satisfying the following boundary value conditions:

$$
(S(-\infty), I(-\infty))^{T}=E_{1}, \quad(S(+\infty), I(+\infty))^{T}=E_{2},
$$

where $E_{1}, E_{2}$ are the equilibrium points of model (3).

This paper is arranged as follows. In Section 2, we construct a pair of ordered upper-lower solutions of model (3) and establish the uniqueness and strict monotonicity of the traveling wave solutions.

\section{Existence and Asymptotic Decay Rates}

In this section, we will establish the existence of traveling wave solutions of model (3) by constructing a pair of ordered upper-lower solutions. The definition of the upper solution and the lower solution is standard. We assume that the inequality between two vectors throughout this paper is componentwise.

Setting

$$
\widehat{S}=\frac{R_{d}-1}{R_{d}}-S, \quad \widehat{I}=I,
$$

then model (3) can be written as

$$
\begin{aligned}
& \frac{\partial \widehat{S}}{\partial t}=-\nu R_{d}\left(\frac{R_{d}-1}{R_{d}}-\widehat{S}+\widehat{I}\right)\left[1-\left(\frac{R_{d}-1}{R_{d}}-\widehat{S}+\widehat{I}\right)\right] \\
&+R_{0} \frac{\left(\left(R_{d}-1\right) / R_{d}-\widehat{S}\right) \widehat{I}}{\left(R_{d}-1\right) / R_{d}-\widehat{S}+\widehat{I}}+v\left(\frac{R_{d}-1}{R_{d}}-\widehat{S}\right)+d \frac{\partial^{2} \widehat{S}}{\partial x^{2}}, \\
& \frac{\partial \widehat{I}}{\partial t}=R_{0} \frac{\left(\left(R_{d}-1\right) / R_{d}-\widehat{S}\right) \widehat{I}}{\left(R_{d}-1\right) / R_{d}-\widehat{S}+\widehat{I}}-\widehat{I}+d \frac{\partial^{2} \widehat{I}}{\partial x^{2}}, \\
&(\widehat{S}, \widehat{I})^{T}(-\infty)=(0,0)^{T}, \quad(\widehat{S}, \widehat{I})^{T}(+\infty)=\left(\widehat{S}^{*}, \widehat{I}^{*}\right)^{T} .
\end{aligned}
$$

For model (3), the equilibria are $E_{1}=\left(\left(R_{d}-1\right) / R_{d}, 0\right)$ and $E_{2}=\left(S^{*}, I^{*}\right)$, where

$$
S^{*}=\frac{\nu R_{0} R_{d}-R_{0}-\nu+1}{\nu R_{0}^{2} R_{d}}, \quad I^{*}=\left(R_{0}-1\right) S^{*},
$$

and for model (7), the equilibria are $\widehat{E_{1}}=(0,0)$ and $\widehat{E_{2}}=$ $\left(\widehat{S}^{*}, \widehat{I}^{*}\right)$, where

$$
\begin{gathered}
\widehat{S}^{*}=\frac{\left(R_{0}-1\right)\left(\nu R_{0} R_{d}-\nu R_{0}-v+1\right)}{\nu R_{0}^{2} R_{d}}, \\
\widehat{I}^{*}=\frac{\left(R_{0}-1\right)\left(\nu R_{0} R_{d}-R_{0}-\nu+1\right)}{\nu R_{0}^{2} R_{d}} .
\end{gathered}
$$

Obviously,

$$
\begin{gathered}
\widehat{I}^{*}-\widehat{S}^{*}=\frac{(\nu-1)\left(R_{0}-1\right)}{\nu R_{0} R_{d}}, \\
\widehat{S}^{*}=\frac{R_{d}-1}{R_{d}}-S^{*}, \quad \widehat{I}^{*}=I^{*} .
\end{gathered}
$$

For simplicity, we define the following functions and constants:

$$
\begin{gathered}
\alpha_{0}=\frac{R_{d}-1}{R_{d}}, \quad \phi(I)=\alpha_{0} \widehat{I}^{*}+\left(\widehat{I}^{*}-\widehat{S}^{*}\right) I ; \\
\beta_{0}=\alpha_{0}\left(\widehat{I}^{*}\right)^{2} \frac{R_{0}-1}{R_{0}}\left(R_{0}-\nu+1\right) ; \\
\gamma_{0}=2 \nu\left(R_{d}-1\right)\left(\widehat{I}^{*}-\widehat{S}^{*}\right)-\left[\nu \widehat{I}^{*}+\left(R_{0}-1\right) \widehat{S}^{*}\right] ; \\
\psi(I)=\nu R_{d}\left(\widehat{I}^{*}-\widehat{S}^{*}\right)^{2} I^{2}+\gamma_{0} \widehat{I}^{*} I+\beta_{0} ; \\
\eta_{0}=-\frac{\gamma_{0} \widehat{I}^{*}}{2 \nu R_{d}\left(\widehat{I}^{*}-\widehat{S}^{*}\right)^{2}} ; \\
\varphi(I)=1, \quad I>0 ; \quad \varphi(I)=-1, \quad I \leq 0 ; \\
B=\frac{\widehat{I}^{*}}{2}\left[1+\varphi\left(\frac{\widehat{I}^{*}}{2}-\eta_{0}\right)\right] .
\end{gathered}
$$

And we will always assume the following hypotheses throughout the rest of this paper: 
$[H 1]$

$$
\begin{gathered}
R_{0}>1, \quad 1<R_{d}<\frac{2 R_{0}^{2}+2 R_{0}-2}{3 R_{0}^{2}-2 R_{0}}, \\
\max \left\{\frac{27 R_{0}\left(R_{d}-1\right)^{2}}{R_{d}^{3}}, \frac{R_{0}-1}{R_{0} R_{d}-1}\right\} \\
<\nu<\frac{-1}{R_{0} R_{d}-R_{0}-1} .
\end{gathered}
$$

$[\mathrm{H} 2]$

$$
\begin{gathered}
v \geq \max \left\{\frac{R_{0}}{2-R_{d}}, \frac{R_{0}^{3}-2 R_{0}^{2}+4 R_{0}-2}{2 R_{0}^{2}+2 R_{0}-2-\left(3 R_{0}^{2}-2 R_{0}\right) R_{d}}\right\}, \\
\psi(B) \leq 0 .
\end{gathered}
$$

Then we can obtain the following.

Lemma 1. If [H1] holds, then $E_{2}$ and $\widehat{E_{2}}$ are endemic points of model (3) and model (7), respectively.

Lemma 2. For model (7), if [H1] holds, then $\widehat{E_{1}}$ is unstable, and $\widehat{E_{2}}$ is stable.

For the sake of convenience, let $x=\sqrt{d} \tilde{x}$. For simplicity, we still use the variables $S, I$, and $x$ instead of $\widehat{S}, \widehat{I}$, and $\tilde{x}$, respectively, then model (7) could be rewritten as

$$
\begin{gathered}
\frac{\partial S}{\partial t}=-\nu R_{d}\left(\alpha_{0}-S+I\right)\left[1-\left(\alpha_{0}-S+I\right)\right] \\
+R_{0} \frac{\left(\alpha_{0}-S\right) I}{\alpha_{0}-S+I}+\nu\left(\alpha_{0}-S\right)+\frac{\partial^{2} S}{\partial x^{2}}, \\
\frac{\partial I}{\partial t}=R_{0} \frac{\left(\alpha_{0}-S\right) I}{\alpha_{0}-S+I}-I+\frac{\partial^{2} I}{\partial x^{2}},
\end{gathered}
$$

$$
(S, I)^{T}(-\infty)=(0,0)^{T}, \quad(S, I)^{T}(+\infty)=\left(\widehat{S}^{*}, \widehat{I}^{*}\right)^{T} .
$$

Following the definition of quasi-monotonicity [17], we can obtain the following results.

Lemma 3. Model (14) is a quasi-monotone decreasing system in $(S, I) \in\left[\widehat{E_{1}}, \widehat{E_{2}}\right]$.

Proof. Let

$$
\begin{gathered}
F_{1}(S, I)=-\nu R_{d}\left(\alpha_{0}-S+I\right)\left[1-\left(\alpha_{0}-S+I\right)\right] \\
+R_{0} \frac{\left(\alpha_{0}-S\right) I}{\alpha_{0}-S+I}+\nu\left(\alpha_{0}-S\right), \\
F_{2}(S, I)=R_{0} \frac{\left(\alpha_{0}-S\right) I}{\alpha_{0}-S+I}-I .
\end{gathered}
$$

From [17], we can know that the functions $F_{1}(S, I)$ and $F_{2}(S, I)$ are said to possess a quasi-monotone nonincreasing system, if the sign of $\partial F_{1}(S, I) / \partial I$ and $\partial F_{2}(S, I) / \partial S$ are both nonpositive.

Since

$$
\begin{gathered}
\frac{\partial F_{2}(S, I)}{\partial S}=-R_{0}\left(\frac{I}{\alpha_{0}-S+I}\right)^{2} \leq 0 \\
\frac{\partial F_{1}(S, I)}{\partial I}=R_{0}\left(\frac{\alpha_{0}-S}{\alpha_{0}-S+I}\right)^{2}+2 \nu R_{d}\left(\alpha_{0}-S+I\right)-\nu R_{d}, \\
\frac{\partial}{\partial S}\left(\frac{\partial F_{1}(S, I)}{\partial I}\right)=-2 \nu R_{d}-2 R_{0} \frac{\left(\alpha_{0}-S\right) I}{\left(\alpha_{0}-S+I\right)^{3}} \leq-2 \nu R_{d}<0 .
\end{gathered}
$$

Then,

$$
\frac{\partial F_{1}(S, I)}{\partial I} \leq R_{0}\left(\frac{\alpha_{0}}{\alpha_{0}+I}\right)^{2}+2 \nu R_{d}\left(\alpha_{0}+I\right)-v R_{d} .
$$

Let

$$
G(z)=\frac{\alpha_{0}^{2} R_{0}}{z^{2}}+2 v R_{d} z-v R_{d}, \quad z \in\left[\alpha_{0}, \alpha_{0}+\widehat{I^{*}}\right],
$$

then

$$
G^{\prime}(z)=2 \nu R_{d}-\frac{2 \alpha_{0}^{2} R_{0}}{z^{3}}=0,
$$

obviously, $z^{*}=\sqrt[3]{\alpha_{0}^{2} R_{0} / \nu R_{d}}$ is the unique real root of $G^{\prime}(z)$.

Since $v>27 R_{0}\left(R_{d}-1\right)^{2} / R_{d}^{3}$, consider $\alpha_{0}=\left(R_{d}-1\right) / R_{d}$, then we can get

$$
G\left(z^{*}\right)=\frac{\left(\alpha_{0}^{2} R_{0}\right)^{2 / 3}\left[\left(27 \alpha_{0}^{2} R_{0}\right)^{1 / 3}-\left(\nu R_{d}\right)^{1 / 3}\right]}{\left(z^{*}\right)^{2}}<0 .
$$

And

$$
\lim _{z \rightarrow 0^{+}} G(z)=\lim _{z \rightarrow+\infty} G(z)=+\infty ;
$$

hence, $G(z)$ has two positive roots.

Since $v \geq R_{0} /\left(2-R_{d}\right)$, thus $G\left(\alpha_{0}\right)=R_{0}+v R_{d}-2 v \leq 0$.

According to conditions [H1] and [H2], we can get

$$
\begin{aligned}
G\left(\alpha_{0}+\widehat{I^{*}}\right)= & \nu R_{d}-2 \nu+2 v R_{d} \widehat{I^{*}}+R_{0}\left(\frac{\alpha_{0}}{\alpha_{0}+\widehat{I^{*}}}\right)^{2} \\
< & \nu R_{d}-2 \nu+2 v R_{d} \widehat{I^{*}}+R_{0} \\
= & \frac{\left(3 R_{0}^{2} R_{d}-2 R_{0}^{2}-2 R_{0} R_{d}-2 R_{0}+2\right) \nu}{R_{0}^{2}} \\
& +\frac{\left(R_{0}^{3}-2 R_{0}^{2}+4 R_{0}-2\right)}{R_{0}^{2}} \\
\leq & 0 .
\end{aligned}
$$

Then, $G\left(\left[\alpha_{0}, \alpha_{0}+\widehat{I^{*}}\right]\right) \leq 0$. Hence, $\partial F_{1}(S, I) / \partial I \leq 0$.

That is to say, model (14) is a quasi-monotone system in $(S, I) \in\left[\widehat{E_{1}}, \widehat{E_{2}}\right]$. 
Since the traveling wave solution of model (14) has the following form

$S(\xi)=S(x+c t), \quad I(\xi)=I(x+c t), \quad \xi=x+c t, \quad c>0 ;$

substituting (23) into model (14), we can get the following model:

$$
\begin{gathered}
S^{\prime \prime}-c S^{\prime}-v R_{d}\left(\alpha_{0}-S+I\right)\left[1-\left(\alpha_{0}-S+I\right)\right] \\
+R_{0} \frac{\left(\alpha_{0}-S\right) I}{\alpha_{0}-S+I}+v\left(\alpha_{0}-S\right)=0 \\
I^{\prime \prime}-c I^{\prime}+R_{0} \frac{\left(\alpha_{0}-S\right) I}{\alpha_{0}-S+I}-I=0, \\
(S, I)^{T}(-\infty)=(0,0)^{T}, \quad(S, I)^{T}(+\infty)=\left(\widehat{S}^{*}, \widehat{I}^{*}\right)^{T} .
\end{gathered}
$$

Obviously, we can know the following.

Remark 4. Model (24) is also a quasi-monotone system in $(S, I) \in\left[\widehat{E_{1}}, \widehat{E_{2}}\right]$.

Now we establish the existence of traveling wave solutions of model (24) through monotone iteration of a pair of smooth upper and lower solutions. Following [17], we give the definitions of the upper and lower solutions of model (24) as follows, respectively.

Definition 5. A smooth function $(\bar{S}(\xi), \bar{I}(\xi))^{T}(\xi \in \mathbb{R})$ is an upper solution of model (24) if its derivatives $\left(\bar{S}^{\prime}, \bar{I}^{\prime}\right)^{T}$ and $\left(\bar{S}^{\prime \prime}, \bar{I}^{\prime \prime}\right)$ are continuous on $\mathbb{R}$, and $(\bar{S}, \bar{I})^{T}$ satisfies

$$
\begin{gathered}
S^{\prime \prime}-c S^{\prime}-\nu R_{d}\left(\alpha_{0}-S+I\right)\left[1-\left(\alpha_{0}-S+I\right)\right] \\
+R_{0} \frac{\left(\alpha_{0}-S\right) I}{\alpha_{0}-S+I}+\nu\left(\alpha_{0}-S\right) \leq 0, \\
I^{\prime \prime}-c I^{\prime}+R_{0} \frac{\left(\alpha_{0}-S\right) I}{\alpha_{0}-S+I}-I \leq 0,
\end{gathered}
$$

with the following boundary value conditions

$$
\left(\begin{array}{l}
S \\
I
\end{array}\right)(-\infty)=\left(\begin{array}{l}
0 \\
0
\end{array}\right), \quad\left(\begin{array}{l}
S \\
I
\end{array}\right)(+\infty) \geq\left(\begin{array}{l}
\widehat{S}^{*} \\
\widehat{I}^{*}
\end{array}\right) .
$$

Definition 6. A smooth function $(\underline{S}(\xi), \underline{I}(\xi))^{T}(\xi \in \mathbb{R})$ is a lower solution of model (24) if its derivatives $\left(\underline{S}^{\prime}, \underline{I}^{\prime}\right)^{T}$ and $\left(\underline{S}^{\prime \prime}, \underline{I}^{\prime \prime}\right)$ are continuous on $\mathbb{R}$, and $(\underline{S}, \underline{I})^{T}$ satisfies

$$
\begin{gathered}
S^{\prime \prime}-c S^{\prime}-\nu R_{d}\left(\alpha_{0}-S+I\right)\left[1-\left(\alpha_{0}-S+I\right)\right] \\
+R_{0} \frac{\left(\alpha_{0}-S\right) I}{\alpha_{0}-S+I}+\nu\left(\alpha_{0}-S\right) \geq 0, \\
I^{\prime \prime}-c I^{\prime}+R_{0} \frac{\left(\alpha_{0}-S\right) I}{\alpha_{0}-S+I}-I \geq 0,
\end{gathered}
$$

with the following boundary value conditions

$$
\left(\begin{array}{l}
S \\
I
\end{array}\right)(-\infty)=\left(\begin{array}{l}
0 \\
0
\end{array}\right), \quad\left(\begin{array}{l}
S \\
I
\end{array}\right)(+\infty) \leq\left(\begin{array}{c}
\widehat{S}^{*} \\
\widehat{I}^{*}
\end{array}\right) .
$$

The construction of the smooth upper-lower solution pair is based on the solution of the following KPP equation:

$$
\begin{gathered}
w^{\prime \prime}-c w^{\prime}+f(w)=0, \\
w(-\infty)=0, \quad w(+\infty)=b,
\end{gathered}
$$

where $f \in C^{2}([0, b])$ and $f>0$ in the open interval $(0, b)$ with $f(0)=f(b)=0, f^{\prime}(0)=a_{1}>0$, and $f^{\prime}(b)=-b_{1}<0$ [15]. First, let us recall the following result.

Lemma 7 (see $[1,15]$ ). Corresponding to every $c \geq 2 \sqrt{a_{1}}$, model (29) has a unique (up to a translation of the origin) monotonically increasing traveling wave solution $w(\xi)$ for $\xi \epsilon$ $R$. The traveling wave solution $w$ has the following asymptotic behaviors.

(i) For the wave solution with noncritical speed $c>2 \sqrt{a_{1}}$, one has

$$
\begin{aligned}
w(\xi)= & a_{\omega} e^{\left(\left(c-\sqrt{c^{2}-4 a_{1}}\right) / 2\right) \xi} \\
& +o\left(e^{\left(\left(c-\sqrt{c^{2}-4 a_{1}}\right) / 2\right) \xi}\right) \text { as } \xi \longrightarrow-\infty \\
w(\xi)= & b-b_{\omega} e^{\left(\left(c-\sqrt{c^{2}+4 b_{1}}\right) / 2\right) \xi} \\
& +o\left(e^{\left(\left(c-\sqrt{c^{2}+4 b_{1}}\right) / 2\right) \xi}\right) \text { as } \xi \longrightarrow+\infty
\end{aligned}
$$

where $a_{\omega}$ and $b_{\omega}$ are positive constants.

(ii) For the wave with critical speed $c=2 \sqrt{a_{1}}$, one has

$$
\begin{aligned}
& w(\xi)=\left(a_{c}+\right.\left.d_{c} \xi\right) e^{\sqrt{a_{1}} \xi}+o\left(\xi e^{\sqrt{a_{1}} \xi}\right) \quad \text { as } \xi \longrightarrow-\infty \\
& \begin{aligned}
w(\xi)= & b-b_{c} e^{\left(\sqrt{a_{1}}-\sqrt{a_{1}+b_{1}}\right) \xi} \\
& +o\left(e^{\left(\sqrt{a_{1}}-\sqrt{a_{1}+b_{1}}\right) \xi}\right) \text { as } \xi \longrightarrow+\infty
\end{aligned}
\end{aligned}
$$

where the constant $d_{c}$ is negative, $b_{c}$ is positive, and $a_{c} \in R$.

For constructing the upper solution of the model (24), we start with the following model:

$$
\begin{gathered}
I^{\prime \prime}-c I^{\prime}+I\left(\widehat{I}^{*}-I\right) \frac{\alpha_{0}\left(R_{0}-1\right)}{\alpha_{0} \widehat{I}^{*}+\left(\widehat{I}^{*}-\widehat{S}^{*}\right) I}=0, \\
I(-\infty)=0, \quad I(+\infty)=\widehat{I}^{*}
\end{gathered}
$$

Define $f(I)=I\left(\widehat{I}^{*}-I\right)\left(\alpha_{0}\left(R_{0}-1\right) / \phi(I)\right), I \in\left[0, \widehat{I}^{*}\right]$, one can verify that all of the following conditions are satisfied:

(i) $f(I)=I\left(\widehat{I}^{*}-I\right)\left(\alpha_{0}\left(R_{0}-1\right) / \phi(I)\right) \in C^{2}\left(\left[0, \widehat{I}^{*}\right]\right)$; 
(ii) $f(I)>0$, for all $I \in\left(0, \widehat{I}^{*}\right)$ and $f(0)=f\left(\widehat{I}^{*}\right)=0$;

(iii) $f^{\prime}(0)=R_{0}-1>0, f^{\prime}\left(\widehat{I}^{*}\right)=-\alpha_{0}\left(R_{0}-1\right)^{2} / R_{0} \widehat{I}^{*}<0$.

From Lemma 7, we know that, for each $c \geq 2 \sqrt{R_{0}-1}$, equation (32) has a unique traveling wave solution $\bar{I}(\xi)$ (up to a translation of the origin), satisfying the given boundary value conditions (26).

Define

$$
\left(\begin{array}{l}
\bar{S}(\xi) \\
\bar{I}(\xi)
\end{array}\right)=\left(\begin{array}{c}
\widehat{S}^{*} \\
\widehat{\bar{I}}_{\bar{I}}^{*}(\xi) \\
\bar{I}(\xi)
\end{array}\right), \quad \xi \in R,
$$

then we can get the following result.

Lemma 8. For each $c \geq 2 \sqrt{R_{0}-1}$, (33) is a smooth upper solution of model (24).

Proof. On the boundary,

$$
\left(\begin{array}{l}
\bar{S} \\
\bar{I}
\end{array}\right)(-\infty)=\left(\begin{array}{l}
0 \\
0
\end{array}\right), \quad\left(\begin{array}{l}
\bar{S} \\
\bar{I}
\end{array}\right)(+\infty) \geq\left(\begin{array}{l}
\widehat{S}^{*} \\
\widehat{I}^{*}
\end{array}\right) .
$$

As for the I component, we have

$$
\begin{aligned}
\bar{I}^{\prime \prime}- & c \bar{I}^{\prime}+R_{0} \frac{\left(\alpha_{0}-\bar{S}\right) \bar{I}}{\alpha_{0}-\bar{S}+\bar{I}}-\bar{I} \\
= & -\bar{I}\left(\widehat{I}^{*}-\bar{I}\right) \frac{\alpha_{0}\left(R_{0}-1\right)}{\phi(\bar{I})}+R_{0} \frac{\left(\alpha_{0}-\bar{S}\right) \bar{I}}{\alpha_{0}-\bar{S}+\bar{I}}-\bar{I} \\
= & -\bar{I}\left(\widehat{I}^{*}-\bar{I}\right) \frac{\alpha_{0}\left(R_{0}-1\right)}{\phi(\bar{I})}+R_{0} \frac{\alpha_{0} \widehat{I}^{*}-\widehat{S}^{*} \bar{I}}{\phi(\bar{I})} \bar{I}-\bar{I} \\
= & -\bar{I}\left(\widehat{I}^{*}-\bar{I}\right) \frac{\alpha_{0}\left(R_{0}-1\right)}{\phi(\bar{I})} \\
& +\bar{I} \frac{\alpha_{0}\left(R_{0}-1\right) \widehat{I}^{*}-\left(R_{0} \widehat{S}^{*}-\widehat{S}^{*}+\widehat{I}^{*}\right) \bar{I}}{\phi(\bar{I})} \\
= & -\bar{I}\left(\widehat{I}^{*}-\bar{I}\right) \frac{\alpha_{0}\left(R_{0}-1\right)}{\phi(\bar{I})} \\
& +\bar{I} \frac{\alpha_{0}\left(R_{0}-1\right) \widehat{I}^{*}-\alpha_{0}\left(R_{0}-1\right) \bar{I}}{\phi(\bar{I})} \\
= & 0 .
\end{aligned}
$$

As for the $S$ component, since $v>1$, then $\widehat{I}^{*}-\widehat{S}^{*}=(\nu-$ 1) $\left(R_{0}-1\right) / \nu R_{0} R_{d}>0$. And

(i) if $\eta_{0}<\widehat{I}^{*} / 2$, then $\max _{\xi \in\left[0, \hat{I}^{*}\right]} \psi(\bar{I})=\psi\left(\widehat{I}^{*}\right)=\psi(B)$;

(ii) if $\eta_{0} \geq \widehat{I}^{*} / 2$, then $\max _{\xi \in\left[0, \widehat{I}^{*}\right]} \psi(\bar{I})=\psi(0)=\psi(B)$.
Thus we can get:

$$
\begin{aligned}
\bar{S}^{\prime \prime}- & c \bar{S}^{\prime}-v R_{d}\left(\alpha_{0}-\bar{S}+\bar{I}\right)\left[1-\left(\alpha_{0}-\bar{S}+\bar{I}\right)\right] \\
& +R_{0} \frac{\left(\alpha_{0}-\bar{S}\right) \bar{I}}{\alpha_{0}-\bar{S}+\bar{I}}+\nu\left(\alpha_{0}-\bar{S}\right) \\
= & \frac{\widehat{S}^{*}}{\widehat{I}^{*}}\left(\bar{I}^{\prime \prime}-c \bar{I}^{\prime}\right)-\nu R_{d}\left(\alpha_{0}-\bar{S}+\bar{I}\right)\left[1-\left(\alpha_{0}-\bar{S}+\bar{I}\right)\right] \\
& +R_{0} \frac{\left(\alpha_{0}-\bar{S}\right) \bar{I}}{\alpha_{0}-\bar{S}+\bar{I}}+v\left(\alpha_{0}-\bar{S}\right) \\
= & \frac{\widehat{S}^{*}}{\widehat{I}^{*}}\left(-R_{0} \frac{\left(\alpha_{0}-\bar{S}\right) \bar{I}}{\alpha_{0}-\bar{S}+\bar{I}}+\bar{I}\right)-v R_{d}\left(\alpha_{0}-\bar{S}+\bar{I}\right) \\
& \times\left[1-\left(\alpha_{0}-\bar{S}+\bar{I}\right)\right]+R_{0} \frac{\left(\alpha_{0}-\bar{S}\right) \bar{I}}{\alpha_{0}-\bar{S}+\bar{I}}+\nu\left(\alpha_{0}-\bar{S}\right) \\
= & \left(1-\frac{\widehat{S}^{*}}{\widehat{I}^{*}}\right) R_{0} \frac{\left(\alpha_{0}-\bar{S}\right) \bar{I}}{\alpha_{0}-\bar{S}+\bar{I}}+\frac{\widehat{S}^{*}}{\widehat{I}^{*}} \bar{I}-\nu R_{d}\left(\alpha_{0}-\bar{S}+\bar{I}\right) \\
& \times\left[1-\left(\alpha_{0}-\bar{S}+\bar{I}\right)\right]+\nu\left(\alpha_{0}-\bar{S}\right) \\
= & \frac{\widehat{I}^{*}-\widehat{S}^{*}}{\widehat{I}^{*}} R_{0} \frac{\alpha_{0} \widehat{I}^{*}-\widehat{S}^{*} \bar{I}}{\phi(\bar{I})} \bar{I}+\frac{\widehat{S}^{*}}{\widehat{I}^{*}} \bar{I}-v R_{d} \frac{\phi(\bar{I})}{\widehat{I}^{*}}\left[1-\frac{\phi(\bar{I})}{\widehat{I}^{*}}\right] \\
& +\nu \frac{\alpha_{0} \widehat{I}^{*}-\widehat{S}^{*} \bar{I}}{\widehat{I}^{*}} \phi(\bar{I}) \\
& \left(\widehat{I}^{*} \psi(\bar{I})\right. \\
= &
\end{aligned}
$$

Hence, $(\bar{S}, \bar{I})$ forms a smooth upper solution for model (24).

For constructing the lower solution of the model (24), we start with the following model:

$$
\begin{gathered}
I^{\prime \prime}-c I^{\prime}+I\left[\widehat{I}^{*}-(1+\varepsilon) I\right] \frac{\alpha_{0}\left(R_{0}-1\right)}{\alpha_{0} \widehat{I}^{*}+\left(\widehat{I}^{*}-\widehat{S}^{*}\right) I}=0, \\
I(-\infty)=0, \quad I(+\infty)=\frac{\widehat{I}^{*}}{1+\varepsilon} .
\end{gathered}
$$

Define $g(I)=I\left[\widehat{I}^{*}-(1+\varepsilon) I\right]\left(\alpha_{0}\left(R_{0}-1\right) /\left(\alpha_{0} \widehat{I}^{*}+\left(\widehat{I}^{*}-\right.\right.\right.$ $\left.\left.\left.\widehat{S}^{*}\right) I\right)\right), I \in\left[0, \widehat{I}^{*} /(1+\varepsilon)\right]$. One can easily verify that all of the following conditions hold:

(i) $g(I)=I\left[\widehat{I}^{*}-(1+\varepsilon) I\right]\left(\alpha_{0}\left(R_{0}-1\right) /\left(\alpha_{0} \widehat{I}^{*}+\left(\widehat{I}^{*}-\widehat{S}^{*}\right) I\right)\right) \epsilon$ $C^{2}\left(\left[0, \widehat{I}^{*} /(1+\varepsilon)\right]\right)$;

(ii) $g(I)>0$, for all $I \in\left(0, \widehat{I}^{*} /(1+\varepsilon)\right)$ and $g(0)=g\left(\widehat{I}^{*} /(1+\right.$ $\varepsilon))=0$ 
(iii) $g^{\prime}(0)=R_{0}-1>0, g^{\prime}\left(\widehat{I}^{*} /(1+\varepsilon)\right)=-(1+\varepsilon) \alpha_{0}\left(R_{0}-\right.$ 1) $/\left(\varepsilon \alpha_{0}+\left(R_{0} /\left(R_{0}-1\right)\right) \widehat{I}^{*}\right)<0$.

From Lemma 7, we know that, for each fixed $c \geq$ $2 \sqrt{R_{0}-1}$, model (37) has a unique traveling wave solution $\underline{I}(\xi)$ (up to a translation of the origin), satisfying the given boundary value conditions (28).

Define

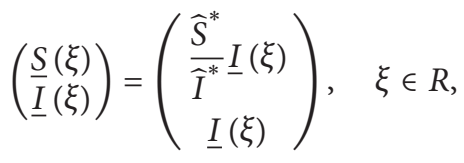

then we have the following result:

Lemma 9. For each fixed $c \geq 2 \sqrt{R_{0}-1}$, (38) is a lower solution of model (24).

Proof. On the boundary,

$$
\left(\begin{array}{l}
\underline{S} \\
\underline{I}
\end{array}\right)(-\infty)=\left(\begin{array}{l}
0 \\
0
\end{array}\right), \quad\left(\frac{\underline{S}}{\underline{I}}\right)(+\infty)=\left(\begin{array}{c}
\frac{\widehat{S}^{*}}{1+\varepsilon} \\
\frac{\widehat{I}^{*}}{1+\varepsilon}
\end{array}\right) \leq\left(\begin{array}{l}
\widehat{S}^{*} \\
\widehat{I}^{*}
\end{array}\right) .
$$

As for the I component, we have

$$
\begin{aligned}
\underline{I}^{\prime \prime} & -c \underline{I}^{\prime}+R_{0} \frac{\left(\alpha_{0}-\underline{S}\right) \underline{I}}{\alpha_{0}-\underline{S}+\underline{I}}-\underline{I} \\
& =-\underline{I}\left[\widehat{I}_{*}-(1+\varepsilon) \underline{I}\right] \frac{\alpha_{0}\left(R_{0}-1\right)}{\phi(\underline{I})}+R_{0} \frac{\left(\alpha_{0}-\underline{S}\right) \underline{I}}{\alpha_{0}-\underline{S}+\underline{I}}-\underline{I} \\
& =-\underline{I}\left[\widehat{I}_{*}-(1+\varepsilon) \underline{I}\right] \frac{\alpha_{0}\left(R_{0}-1\right)}{\phi(\underline{I})}+\underline{I}\left(\widehat{I}^{*}-\underline{I}\right) \frac{\alpha_{0}\left(R_{0}-1\right)}{\phi(\underline{I})} \\
& =\varepsilon(\underline{I})^{2} \frac{\alpha_{0}\left(R_{0}-1\right)}{\phi(\underline{I})} \geq 0 .
\end{aligned}
$$

As for the $S$ component, we have

$$
\begin{aligned}
\underline{S}^{\prime \prime}-c \underline{S}^{\prime}-\nu R_{d}\left(\alpha_{0}-\underline{S}+\underline{I}\right)\left[1-\left(\alpha_{0}-\underline{S}+\underline{I}\right)\right] \\
\quad+R_{0} \frac{\left(\alpha_{0}-\underline{S}\right) \underline{I}}{\alpha_{0}-\underline{S}+\underline{I}}+\nu\left(\alpha_{0}-\underline{S}\right) \\
=\frac{\widehat{S}^{*}}{\widehat{I}^{*}}\left(\underline{I}^{\prime \prime}-c \underline{I}^{\prime}\right)-\nu R_{d}\left(\alpha_{0}-\underline{S}+\underline{I}\right)\left[1-\left(\alpha_{0}-\underline{S}+\underline{I}\right)\right] \\
\quad+R_{0} \frac{\left(\alpha_{0}-\underline{S}\right) \underline{I}}{\alpha_{0}-\underline{S}+\underline{I}}+v\left(\alpha_{0}-\underline{S}\right)
\end{aligned}
$$

$$
\begin{aligned}
= & \frac{\widehat{S}^{*}}{\widehat{I}^{*}}\left\{\left[\varepsilon(\underline{I})^{2} \frac{\alpha_{0}\left(R_{0}-1\right)}{\phi(\underline{I})}\right]-\left[R_{0} \frac{\left(\alpha_{0}-\underline{S}\right) \underline{I}}{\alpha_{0}-\underline{S}+\underline{I}}-\underline{I}\right]\right\} \\
& -\nu R_{d}\left(\alpha_{0}-\underline{S}+\underline{I}\right)\left[1-\left(\alpha_{0}-\underline{S}+\underline{I}\right)\right]+R_{0} \frac{\left(\alpha_{0}-\underline{S}\right) \underline{I}}{\alpha_{0}-\underline{S}+\underline{I}} \\
& +\nu\left(\alpha_{0}-\underline{S}\right) \\
= & \varepsilon \frac{\widehat{S}^{*}}{\widehat{I}^{*}}(\underline{I})^{2} \frac{\alpha_{0}\left(R_{0}-1\right)}{\phi(\underline{I})}+\frac{\left(\widehat{I}^{*}-\widehat{S}^{*}\right) \underline{I} \psi(\underline{I})}{\left(\widehat{I}^{*}\right)^{2} \phi(\underline{I})} \geq 0 .
\end{aligned}
$$

Thus $(\underline{S}, \underline{I})$ forms a smooth lower solution for model (24).

Next, we show that, by shifting the upper solution far enough to the left, then the upper-lower solution in Lemmas 8 and 9 are ordered.

Lemma 10. Let $c \geq 2 \sqrt{R_{0}-1},(\bar{S}, \bar{I})^{T}$ and $(\underline{S}, \underline{I})^{T}$ be the upper solution and the lower solution defined in (33) and (38), then there exists a positive number $r$, such that $(\bar{S}, \bar{I})^{T}(\xi+r) \geq$ $(\underline{S}, \underline{I})^{T}(\xi)$ for all $\xi \in R$.

Proof. Our proof is only for $c>2 \sqrt{R_{0}-1}$, and the proof for the case of $c=2 \sqrt{R_{0}-1}$ is similar to it.

First, we derive the asymptotic behaviors of the upper solution and the lower solution at infinities.

According to Lemma 7, when $\xi \rightarrow-\infty$, we can obtain:

$$
\begin{aligned}
& \left(\begin{array}{c}
\bar{S} \\
\bar{I}
\end{array}\right)(\xi)=\left(\begin{array}{c}
\frac{\widehat{S}^{*}}{\widehat{I}^{*}} A_{1} \\
A_{1}
\end{array}\right) e^{\left(\left(c-\sqrt{\left.c^{2}-4\left(R_{0}-1\right)\right)} / 2\right) \xi\right.} \\
& +o\left(e^{\left(\left(c-\sqrt{\left.c^{2}-4\left(R_{0}-1\right)\right)} / 2\right) \xi\right.}\right), \\
& \left(\frac{S}{I}\right)(\xi)=\left(\begin{array}{c}
\widehat{S}^{*} \\
\widehat{I}^{*} B_{1} \\
B_{1}
\end{array}\right) e^{\left(\left(c-\sqrt{c^{2}-4\left(R_{0}-1\right)}\right) / 2\right) \xi} \\
& +o\left(e^{\left(\left(c-\sqrt{c^{2}-4\left(R_{0}-1\right)}\right) / 2\right) \xi}\right) .
\end{aligned}
$$

And let $\sigma_{0}=(1 / 2)(c)-$
$\left.\sqrt{c^{2}+4\left(\alpha_{0}\left(R_{0}-1\right)^{2} / R_{0} \widehat{I}^{*}\right.}\right)<0, \delta_{0}=(1 / 2)(c-$
$\left.\sqrt{c^{2}+4\left((1+\varepsilon) \alpha_{0}\left(R_{0}-1\right) /\left(\varepsilon \alpha_{0}+\left(R_{0} /\left(R_{0}-1\right)\right) \widehat{I}^{*}\right)\right.}\right)<0$,
when $\xi \rightarrow+\infty$, we can get

$$
\begin{aligned}
& \left(\begin{array}{l}
\bar{S} \\
\bar{I}
\end{array}\right)(\xi)=\left(\begin{array}{c}
\widehat{S}^{*} \\
\widehat{I}^{*}
\end{array}\right)-\left(\begin{array}{c}
\frac{\widehat{S}^{*}}{\widehat{I}^{*}} A_{2} \\
A_{2}
\end{array}\right) e^{\sigma_{0} \xi}+o\left(e^{\sigma_{0} \xi}\right), \\
& \left(\frac{\underline{S}}{\underline{I}}\right)(\xi)=\frac{1}{1+\varepsilon}\left(\begin{array}{c}
\widehat{S}^{*} \\
\widehat{I}^{*}
\end{array}\right)-\left(\begin{array}{c}
\frac{\widehat{S}^{*}}{\widehat{I}^{*}} B_{2} \\
B_{2}
\end{array}\right) e^{\delta_{0} \xi}+o\left(e^{\delta_{0} \xi}\right),
\end{aligned}
$$

where, $A_{1}, A_{2}, B_{1}, B_{2}$ are all positive constants. 
Since for any $\widetilde{r}>0, \bar{I}^{\widetilde{r}}(\xi) \equiv \bar{I}(\xi+\widetilde{r})$ is also a solution of model (32). Thus, $\left(\bar{S}^{\widetilde{r}}, \bar{I}^{\widetilde{r}}\right)^{T}(\xi)$ is an upper solution of model (24). So, according to Lemma 7 , when $\xi \rightarrow-\infty$, we can get:

$$
\begin{aligned}
\left(\begin{array}{c}
\bar{S}^{\widetilde{r}} \\
\bar{I}^{\widetilde{r}}
\end{array}\right)(\xi)= & \left(\begin{array}{c}
\widehat{S}^{*} \\
\widehat{I}^{*} \\
A_{1}
\end{array}\right) e^{\left(\left(c-\sqrt{c^{2}-4\left(R_{0}-1\right)}\right) / 2\right) \widetilde{r}} e^{\left(\left(c-\sqrt{\left.c^{2}-4\left(R_{0}-1\right)\right)} / 2\right) \xi\right.} \\
& +o\left(e^{\left(\left(c-\sqrt{c^{2}-4\left(R_{0}-1\right)}\right) / 2\right) \xi}\right) .
\end{aligned}
$$

Since $\left(c-\sqrt{c^{2}-4\left(R_{0}-1\right)}\right) / 2>0$, we can choose a large enough number $\tilde{r} \gg 0$, such that

$$
\left(\begin{array}{c}
\frac{\widehat{S}^{*}}{\widehat{I}^{*}} A_{1} \\
A_{1}
\end{array}\right) e^{\left(\left(c-\sqrt{c^{2}-4\left(R_{0}-1\right)}\right) / 2\right) \widetilde{r}}>\left(\begin{array}{c}
\widehat{S}^{*} \\
\widehat{I}^{*} \\
B_{1}
\end{array}\right),
$$

hence, there exists a large number $N_{1} \gg 1$, such that

$$
\left(\begin{array}{l}
\bar{S}^{\widetilde{r}}(\xi) \\
\widetilde{I}^{r}(\xi)
\end{array}\right)>\left(\begin{array}{l}
\underline{S}(\xi) \\
\underline{I}(\xi)
\end{array}\right), \quad \xi \in\left(-\infty,-N_{1}\right] .
$$

By using a similar argument as above, there exists a large enough number $N_{2} \gg 1$, such that

$$
\left(\begin{array}{l}
\bar{S}^{\widetilde{S}}(\xi) \\
\bar{I}^{\widetilde{T}}(\xi)
\end{array}\right)>\left(\begin{array}{l}
\underline{S}(\xi) \\
\underline{I}(\xi)
\end{array}\right), \quad \xi \in\left[N_{2},+\infty\right) .
$$

Second, we show that

$$
\left(\begin{array}{c}
\widetilde{S}^{\widetilde{r}}(\xi) \\
\widetilde{I}^{r}(\xi)
\end{array}\right)>\left(\begin{array}{l}
\underline{S}(\xi) \\
\underline{I}(\xi)
\end{array}\right), \quad \xi \in\left[-N_{1}, N_{2}\right] .
$$

We deal with such two possible cases:

Case 1. If

$$
\left(\begin{array}{c}
\widetilde{S}^{\tilde{r}}(\xi) \\
\tilde{I}^{\tilde{r}}(\xi)
\end{array}\right)>\left(\begin{array}{l}
\underline{S}(\xi) \\
\underline{I}(\xi)
\end{array}\right), \quad \xi \in\left[-N_{1}, N_{2}\right],
$$

then, the proof is completed.

Case 2. If there exists a point $\xi_{0} \in\left(-N_{1}, N_{2}\right)$, such that

$$
\left(\begin{array}{l}
\bar{S}^{\tilde{S}}\left(\xi_{0}\right) \\
\bar{I}^{\tilde{T}}\left(\xi_{0}\right)
\end{array}\right) \leq\left(\begin{array}{l}
\underline{S}\left(\xi_{0}\right) \\
\underline{I}\left(\xi_{0}\right)
\end{array}\right)
$$

satisfying $\bar{S}^{\widetilde{r}}\left(\xi_{0}\right)<\underline{S}\left(\xi_{0}\right)$ or $\bar{I}^{\widetilde{r}}\left(\xi_{0}\right)<\underline{I}\left(\xi_{0}\right)$.

In this case, we use the Sliding Domain method [15].

Step 1. we shift $\left(\bar{S}^{\tilde{r}}, \bar{I}^{\widetilde{r}}\right)^{T}$ to the left by increasing the number $\tilde{r}$ until finding a new number $r_{1}>\widetilde{r}$ such that $\left(\bar{S}^{r_{1}}, \bar{I}^{r_{1}}\right)^{T}>$ $(\underline{S}, \underline{I})^{T}$ on the smaller interval $\left[-N_{1}, N_{2}-\left(r_{1}-\widetilde{r}\right)\right]$.

Step 2. we shift $\left(\bar{S}^{r_{1}}, \bar{I}^{r_{1}}\right)^{T}$ back to the right by decreasing $r_{1}$ to a smaller number $\tilde{r}<r_{2}<r_{1}$ such that one of the branches of the upper solution touches its counterpart of the lower solution at some point $\xi_{1}$ in the interval $\left(-N_{1}+\right.$ $\left.\left(r_{1}-r_{2}\right), N_{2}-\left(r_{1}-\tilde{r}\right)\right)$. On the endpoints of the interval $\left(-N_{1}+\left(r_{1}-r_{2}\right), N_{2}-\left(r_{1}-\widetilde{r}\right)\right)$, we still have $\left(\bar{S}^{r_{2}}, \bar{I}^{r_{2}}\right)^{T}>(\underline{S}, \underline{I})^{T}$.

Let $\vec{W}(\xi)=\left(\bar{S}^{r_{2}}, \bar{I}^{r_{2}}\right)^{T}-(\underline{S}, \underline{I})^{T}$ and $\vec{F}=\left(F_{1}, F_{2}\right)^{T}$, where

$$
\begin{gathered}
F_{1}=-\nu R_{d}\left(\alpha_{0}-S+I\right)\left[1-\left(\alpha_{0}-S+I\right)\right] \\
+R_{0} \frac{\left(\alpha_{0}-S\right) I}{\alpha_{0}-S+I}+\nu\left(\alpha_{0}-S\right) \\
F_{2}=R_{0} \frac{\left(\alpha_{0}-S\right) I}{\alpha_{0}-S+I}-I .
\end{gathered}
$$

For $\xi \in\left(-N_{1}+\left(r_{1}-r_{2}\right), N_{2}-\left(r_{1}-\widetilde{r}\right)\right)$, we get that

$\vec{W}^{\prime \prime}-c \vec{W}^{\prime}+\left(\begin{array}{ll}\frac{\partial F_{1}}{\partial S}\left(\underline{S}+\zeta_{1} \omega_{1}, \bar{I}^{r_{2}}\right) & \frac{\partial F_{1}}{\partial I}\left(\bar{S}^{r_{2}}, \underline{I}+\zeta_{2} \omega_{2}\right) \\ \frac{\partial F_{2}}{\partial S}\left(\underline{S}+\zeta_{3} \omega_{1}, \bar{I}^{r_{2}}\right) & \frac{\partial F_{2}}{\partial I}\left(\bar{S}^{r_{2}}, \underline{I}+\zeta_{4} \omega_{2}\right)\end{array}\right) \vec{W}$ $=0$,

where $\zeta_{i} \in[0,1], i=1,2,3,4$. Since the above model is monotone and the cube $\left[(0,0),\left(\widehat{S}^{*}, \widehat{I}^{*}\right)\right]$ is convex, thus we can deduce by Maximum Principle that $\vec{W}>0$ for $\xi \in$ $\left[-N_{1}+\left(r_{1}-r_{2}\right), N_{2}-\left(r_{1}-\widetilde{r}\right)\right]$. So $\xi_{1}$ does not exist and we can decrease $r_{2}$ further to $\widetilde{r}$. It is calculated that the point $\xi_{0}$ does not exist either. The proof of this lemma is completed.

To ease the burden of notations, we still use $(\bar{S}, \bar{I})^{T}$ to denote the shifted upper solution as given in Lemma 8. Let

$$
\begin{gathered}
D_{11}=-\frac{R_{0}^{2}+\nu R_{0} R_{d}+\nu R_{0}-4 R_{0}-2 \nu+3}{R_{0}}, \\
D_{12}=\frac{\nu R_{0} R_{d}-2 R_{0}-2 \nu+3}{R_{0}}, \\
D_{21}=-\frac{\left(R_{0}-1\right)^{2}}{R_{0}}, \\
D_{22}=-\frac{R_{0}-1}{R_{0}},
\end{gathered}
$$

$$
\begin{aligned}
& \mu_{1}=\frac{-\left(D_{11}+D_{22}\right)+\sqrt{\left(D_{11}-D_{22}\right)^{2}+4 D_{12} D_{21}}}{2}, \\
& \mu_{2}=\frac{-\left(D_{11}+D_{22}\right)-\sqrt{\left(D_{11}-D_{22}\right)^{2}+4 D_{12} D_{21}}}{2} .
\end{aligned}
$$

With such constructed ordered upper-lower solution pair, we can get the following.

Theorem 11. For $c \geq 2 \sqrt{R_{0}-1}$, model (24) has a unique (up to a translation of the origin) traveling wave solution. The traveling wave solution is strictly increasing and has the following asymptotic properties: 
(i) if $c>2 \sqrt{R_{0}-1}$, when $\xi \rightarrow-\infty$,

$$
\begin{aligned}
\left(\begin{array}{l}
S \\
I
\end{array}\right)(\xi)= & \left(\begin{array}{l}
A_{1} \\
A_{2}
\end{array}\right) e^{\left(\left(c-\sqrt{c^{2}-4\left(R_{0}-1\right)}\right) / 2\right) \xi} \\
& +o\left(e^{\left(\left(c-\sqrt{c^{2}-4\left(R_{0}-1\right)}\right) / 2\right) \xi}\right) .
\end{aligned}
$$

when $\xi \rightarrow+\infty$, and if $\mu_{1} \neq \mu_{2}$, then

$$
\begin{aligned}
\left(\begin{array}{l}
S \\
I
\end{array}\right)(\xi)= & \left(\begin{array}{l}
\widehat{S}^{*} \\
\widehat{I}^{*}
\end{array}\right)-\left(\begin{array}{l}
\overline{A_{1}} \\
\overline{A_{2}}
\end{array}\right) e^{\left(\left(c-\sqrt{c^{2}+4 \mu}\right) / 2\right) \xi} \\
& +o\left(e^{\left(\left(c-\sqrt{c^{2}+4 \mu}\right) / 2\right) \xi}\right),
\end{aligned}
$$

while $\mu_{1}=\mu_{2}$ :

$$
\begin{aligned}
\left(\begin{array}{l}
S \\
I
\end{array}\right)(\xi)= & \left(\begin{array}{l}
\widehat{S}^{*} \\
\widehat{I}^{*}
\end{array}\right)-\left(\begin{array}{l}
\overline{\overline{A_{11}}}+\overline{\overline{A_{12}}} \xi \\
\overline{\overline{A_{21}}}+\overline{\overline{A_{22}}} \xi
\end{array}\right) e^{\left(\left(c-\sqrt{c^{2}+4 \mu}\right) / 2\right) \xi} \\
& +o\left(e^{\left(\left(c-\sqrt{c^{2}+4 \mu}\right) / 2\right) \xi}\right)
\end{aligned}
$$

where, $\mu=\min \left\{\mu_{1}, \mu_{2}\right\}>0, \overline{\overline{A_{11}}}, \overline{\overline{A_{21}}} \in \mathbb{R}, A_{1}, A_{2}, \overline{A_{1}}, \overline{A_{2}}$, $\overline{\overline{A_{12}}}$ and $\overline{\overline{A_{22}}}$ are all positive constants.

(ii) if $c=2 \sqrt{R_{0}-1}$, when $\xi \rightarrow-\infty$,

$$
\left(\begin{array}{l}
S \\
I
\end{array}\right)(\xi)=\left(\begin{array}{l}
B_{11}+B_{12} \xi \\
B_{21}+B_{22} \xi
\end{array}\right) e^{\sqrt{R_{0}-1} \xi}+o\left(\xi e^{\sqrt{R_{0}-1} \xi}\right),
$$

when $\xi \rightarrow+\infty$, and if $\mu_{1} \neq \mu_{2}$, then

$$
\begin{aligned}
\left(\begin{array}{l}
S \\
I
\end{array}\right)(\xi)= & \left(\begin{array}{l}
\widehat{S}^{*} \\
\widehat{I}^{*}
\end{array}\right)-\left(\begin{array}{l}
\overline{B_{11}} \\
\overline{B_{22}}
\end{array}\right) e^{\left(\sqrt{R_{0}-1}-\sqrt{R_{0}-1+\mu}\right) \xi} \\
& +o\left(e^{\left(\sqrt{R_{0}-1}-\sqrt{R_{0}-1+\mu}\right) \xi}\right),
\end{aligned}
$$

while $\mu_{1}=\mu_{2}$,

$$
\begin{aligned}
\left(\begin{array}{l}
S \\
I
\end{array}\right)(\xi)= & \left(\begin{array}{l}
\widehat{S}^{*} \\
\widehat{I}^{*}
\end{array}\right)-\left(\begin{array}{c}
\overline{\overline{B_{11}}}+\overline{\overline{B_{12}}} \xi \\
\overline{\overline{B_{21}}}+\overline{\overline{B_{22}}} \xi
\end{array}\right) e^{\left(\sqrt{R_{0}-1}-\sqrt{R_{0}-1+\mu}\right) \xi} \\
& +o\left(e^{\left(\sqrt{R_{0}-1}-\sqrt{R_{0}-1+\mu}\right) \xi}\right),
\end{aligned}
$$

where $\mu=\min \left\{\mu_{1}, \mu_{2}\right\}>0, B_{12}, B_{22}<0, B_{11}, B_{21}, \overline{\overline{B_{11}}}, \overline{\overline{B_{21}}} \epsilon$ $\mathbb{R}$, and $\overline{B_{11}}, \overline{B_{22}}, \overline{\overline{B_{12}}}, \overline{\overline{B_{22}}}$ are all positive constants.

Proof. From Lemma 3 and Remark 4, we know that model (24) is a quasi-monotone nonincreasing system in $(S, I) \in$ $\left[\widehat{E_{1}}, \widehat{E_{2}}\right]$, and by using the monotone iteration scheme given in $[3,13]$, we can obtain the existence of the solution $(S, I)^{T}$ to the first two equations in model (24) for every $c \geq 2 \sqrt{R_{0}-1}$, which satisfies

$$
\left(\begin{array}{l}
\underline{S}(\xi) \\
\underline{I}(\xi)
\end{array}\right) \leq\left(\begin{array}{l}
S(\xi) \\
I(\xi)
\end{array}\right) \leq\left(\begin{array}{l}
\bar{S}(\xi) \\
\bar{I}(\xi)
\end{array}\right)
$$

According to the above inequality, we can get that, on the boundary, the solution tends to $(0,0)^{T}$ as $\xi \rightarrow-\infty$ and $\left(\widehat{S}^{*}, \widehat{I}^{*}\right)^{T}$ as $\xi \rightarrow+\infty$.

To derive the asymptotic decay rate of the traveling wave solutions as $\xi \rightarrow \pm \infty$, we just let $c>2 \sqrt{R_{0}-1}$ and

$$
U(\xi)=(S(\xi), I(\xi))^{T}, \quad-\infty<\xi<+\infty
$$

be the traveling wave solution of model (24) generated form the monotone iteration, since the case of (ii) $c=2 \sqrt{R_{0}-1}$ is similar to it.

We differentiate model (24) with respect to $\xi$, and note that $U^{\prime}(\xi)=\left(\chi_{1}, \chi_{2}\right)^{T}(\xi)$ satisfies

$$
\begin{aligned}
& \chi_{1}^{\prime \prime}-c \chi_{1}^{\prime}+C_{11}(S, I) \chi_{1}+C_{12}(S, I) \chi_{2}=0, \\
& \chi_{2}^{\prime \prime}-c \chi_{2}^{\prime}+C_{21}(S, I) \chi_{1}+C_{22}(S, I) \chi_{2}=0,
\end{aligned}
$$

where

$$
\begin{gathered}
C_{11}(S, I)=R_{d}\left[1-\left(\alpha_{0}-S+I\right)\right]-\nu R_{d}\left(\alpha_{0}-S+I\right) \\
-\frac{R_{0} I}{\alpha_{0}-S+I}+\frac{R_{0}\left(\alpha_{0}-S\right) I}{\left(\alpha_{0}-S+I\right)^{2}}-\nu, \\
C_{12}(S, I)=-\nu R_{d}\left[1-\left(\alpha_{0}-S+I\right)\right]+\nu R_{d}\left(\alpha_{0}-S+I\right) \\
+\frac{R_{0}\left(\alpha_{0}-S\right)}{\alpha_{0}-S+I}-\frac{R_{0}\left(\alpha_{0}-S\right) I}{\left(\alpha_{0}-S+I\right)^{2}}, \\
C_{21}(S, I)=-\frac{R_{0} I}{\alpha_{0}-S+I}+\frac{R_{0}\left(\alpha_{0}-S\right) I}{\left(\alpha_{0}-S+I\right)^{2}}, \\
C_{22}(S, I)=\frac{R_{0}\left(\alpha_{0}-S\right)}{\alpha_{0}-S+I}-\frac{R_{0}\left(\alpha_{0}-S\right) I}{\left(\alpha_{0}-S+I\right)^{2}}-1 .
\end{gathered}
$$

Now, we study the exponential decay rate of the traveling wave solution as $\xi \rightarrow-\infty$. The asymptotic model of model (62) as $\xi \rightarrow-\infty$ is

$$
\begin{aligned}
& \lambda^{\prime \prime}-c \lambda^{\prime}+E_{11} \lambda+E_{12} \mu=0, \\
& \mu^{\prime \prime}-c \mu^{\prime}+E_{21} \lambda+E_{22} \mu=0,
\end{aligned}
$$

where

$$
\begin{gathered}
E_{11}=-v\left(R_{d}-1\right), \quad E_{12}=v R_{d}+R_{0}-2 v, \\
E_{21}=0, \quad E_{22}=R_{0}-1 .
\end{gathered}
$$

The second equation of model (64) has two independent solutions with the following form:

$$
\begin{aligned}
& \mu^{(1)}(\xi)=e^{\left(\left(c-\sqrt{c^{2}-4\left(R_{0}-1\right)}\right) / 2\right) \xi}, \\
& \mu^{(2)}(\xi)=e^{\left(\left(c+\sqrt{c^{2}-4\left(R_{0}-1\right)}\right) / 2\right) \xi} .
\end{aligned}
$$


Relating the second equation of model (62) with the second equation of model (64), we can deduce that $\chi_{2}(\xi)$ has the following property as $\xi \rightarrow-\infty$ :

$$
\begin{aligned}
\chi_{2}(\xi)= & \alpha[1+o(1)] e^{\left(\left(c-\sqrt{c^{2}-4\left(R_{0}-1\right)}\right) / 2\right) \xi} \\
& +\beta[1+o(1)] e^{\left(\left(c+\sqrt{c^{2}-4\left(R_{0}-1\right)}\right) / 2\right) \xi}
\end{aligned}
$$

for some constants $\alpha$ and $\beta$. Thus, we can obtain that

$$
\begin{aligned}
\chi_{2}(\xi)= & \alpha e^{\left(\left(c-\sqrt{c^{2}-4\left(R_{0}-1\right)}\right) / 2\right) \xi}+\beta e^{\left(\left(c+\sqrt{c^{2}-4\left(R_{0}-1\right)}\right) / 2\right) \xi} \\
& +\Upsilon_{1}(\xi)+\Upsilon_{2}(\xi),
\end{aligned}
$$

where

$$
\begin{gathered}
\lim _{\xi \rightarrow-\infty} \frac{\Upsilon_{1}(\xi)}{e^{\left(\left(c-\sqrt{c^{2}-4\left(R_{0}-1\right)}\right) / 2\right) \xi}}=0, \\
\lim _{\xi \rightarrow-\infty} \frac{\Upsilon_{2}(\xi)}{e^{\left(\left(c+\sqrt{c^{2}-4\left(R_{0}-1\right)}\right) / 2\right) \xi}}=0 .
\end{gathered}
$$

So we obtain that

$$
\begin{aligned}
& \lim _{\xi \rightarrow-\infty} \frac{\chi_{2}(\xi)-\alpha e^{\left(\left(c-\sqrt{c^{2}-4\left(R_{0}-1\right)}\right) / 2\right) \xi}}{e^{\left(\left(c-\sqrt{\left.c^{2}-4\left(R_{0}-1\right)\right)} / 2\right) \xi\right.}} \\
& =\lim _{\xi \rightarrow-\infty} \frac{\Upsilon_{1}(\xi)+\Upsilon_{2}(\xi)+\beta e^{\left(\left(c+\sqrt{c^{2}-4\left(R_{0}-1\right)}\right) / 2\right) \xi}}{e^{\left(\left(c-\sqrt{c^{2}-4\left(R_{0}-1\right)}\right) / 2\right) \xi}} \\
& =\lim _{\xi \rightarrow-\infty} \frac{\Upsilon_{1}(\xi)}{e^{\left(\left(c-\sqrt{c^{2}-4\left(R_{0}-1\right)}\right) / 2\right) \xi}}+\beta \lim _{\xi \rightarrow-\infty} e^{\sqrt{c^{2}-4\left(R_{0}-1\right)} \xi} \\
& \quad+\lim _{\xi \rightarrow-\infty} \frac{\Upsilon_{2}(\xi)}{e^{\left(\left(c+\sqrt{c^{2}-4\left(R_{0}-1\right)}\right) / 2\right) \xi}} \lim _{\xi \rightarrow-\infty} e^{\sqrt{c^{2}-4\left(R_{0}-1\right) \xi}}=0 .
\end{aligned}
$$

Thus, $\chi_{2}(\xi)=\alpha[1+o(1)] e^{\left(\left(c-\sqrt{c^{2}-4\left(R_{0}-1\right)}\right) / 2\right) \xi}$.

Now, we consider the first equation of model (64). We rewrite it as

$$
\lambda^{\prime \prime}-c \lambda^{\prime}-\nu\left(R_{d}-1\right) \lambda=-\left(\nu R_{d}+R_{0}-2 \nu\right) \mu .
$$

One can verify that $\left(c-\sqrt{c^{2}-4\left(R_{0}-1\right)}\right) / 2$ is not a characteristic of

$$
\lambda^{\prime \prime}-c \lambda^{\prime}-v\left(R_{d}-1\right) \lambda=0 .
$$

The above equation has two independent solutions of the following form:

$$
\begin{aligned}
& \lambda^{(1)}(\xi)=e^{\left(\left(c-\sqrt{c^{2}+4 v\left(R_{d}-1\right)}\right) / 2\right) \xi}, \\
& \lambda^{(2)}(\xi)=e^{\left(\left(c+\sqrt{\left.c^{2}+4 v\left(R_{d}-1\right)\right)} / 2\right) \xi\right.} .
\end{aligned}
$$

Thus, when $\xi \rightarrow-\infty, \chi_{1}(\xi)$ has the following property:

$$
\begin{aligned}
\chi_{1}(\xi)= & \bar{\alpha}[1+o(1)] e^{\left(\left(c+\sqrt{c^{2}+4 v\left(R_{d}-1\right)}\right) / 2\right) \xi} \\
& +\bar{\beta}[1+o(1)] e^{\left(\left(c-\sqrt{c^{2}+4 v\left(R_{d}-1\right)}\right) / 2\right) \xi} \\
& +\gamma[1+o(1)] e^{\left(\left(\left(c-\sqrt{c^{2}-4\left(R_{0}-1\right)}\right) / 2\right) \xi\right.}
\end{aligned}
$$

for some constants $\bar{\alpha}, \bar{\beta} ; \gamma \neq 0$. Since $\chi_{1}(-\infty)=0$, thus $\bar{\beta}=0$. So, when $\xi \rightarrow-\infty$, we have the following formula:

$$
\left(\begin{array}{l}
\chi_{1}(\xi) \\
\chi_{2}(\xi)
\end{array}\right)=\left(\begin{array}{l}
\gamma[1+o(1)] e^{\left(\left(c-\sqrt{c^{2}-4\left(R_{0}-1\right)}\right) / 2\right) \xi} \\
\alpha[1+o(1)] e^{\left(\left(c-\sqrt{\left.c^{2}-4\left(R_{0}-1\right)\right)} / 2\right) \xi\right.}
\end{array}\right) .
$$

Then, we study the exponential decay rate of the traveling wave solution as $\xi \rightarrow+\infty$. The asymptotic model of model (62) as $\xi \rightarrow+\infty$ is

$$
\begin{aligned}
& \psi_{1}^{\prime \prime}-c \psi_{1}^{\prime}+D_{11} \psi_{1}+D_{12} \psi_{2}=0 \\
& \psi_{2}^{\prime \prime}-c \psi_{2}^{\prime}+D_{21} \psi_{1}+D_{22} \psi_{2}=0 .
\end{aligned}
$$

By setting $\left(\psi_{i}\right)^{\prime}(\xi)=\widetilde{\psi}_{i}, i=1,2$, we rewrite model (76) as a first order model of ordinary differential equation in the four components $\left(\psi_{1}, \widetilde{\psi}_{1}, \psi_{2}, \widetilde{\psi}_{2}\right)^{T}$ :

$$
\begin{gathered}
\psi_{1}^{\prime}=\widetilde{\psi}_{1}, \\
\widetilde{\psi}_{1}^{\prime}=c \widetilde{\psi}_{1}-D_{11} \psi_{1}-D_{12} \psi_{2}, \\
\psi_{2}^{\prime}=\widetilde{\psi}_{2}, \\
\widetilde{\psi}_{2}^{\prime}=c \widetilde{\psi}_{2}-D_{21} \psi_{1}-D_{22} \psi_{2} .
\end{gathered}
$$

In the case of (i) $\mu_{1} \neq \mu_{2}$, we can obtain that the solution of model (77) has the following form:

$$
\left(\psi_{1}, \widetilde{\psi}_{1}, \psi_{2}, \widetilde{\psi}_{2}\right)^{T}=\sum_{i=1}^{4} c_{i} h_{i} e^{\lambda_{i} \xi},
$$

where

$$
\begin{array}{ll}
\lambda_{1}=\frac{c+\sqrt{c^{2}+4 \mu_{1}}}{2}, & \lambda_{2}=\frac{c-\sqrt{c^{2}+4 \mu_{1}}}{2}, \\
\lambda_{3}=\frac{c+\sqrt{c^{2}+4 \mu_{2}}}{2}, & \lambda_{4}=\frac{c-\sqrt{c^{2}+4 \mu_{2}}}{2},
\end{array}
$$

and $h_{i}(i=1,2,3,4)$ are the eigenvectors of the constant matrix with $\lambda_{i}(i=1,2,3,4)$ as the corresponding eigenvalues, $c_{i}(i=1,2,3,4)$ are arbitrary constants. Since

$$
\lim _{\xi \rightarrow+\infty}\left(\psi_{1}, \widetilde{\psi}_{1}, \psi_{2}, \widetilde{\psi}_{2}\right)^{T}=0,
$$

thus $\left(\psi_{1}, \widetilde{\psi}_{1}, \psi_{2}, \widetilde{\psi}_{2}\right)^{T}=c_{2} h_{2} e^{\lambda_{2} \xi}+c_{4} h_{4} e^{\lambda_{4} \xi}$, so when $\xi \rightarrow$ $+\infty$, we can get that

$$
\begin{aligned}
\left(\begin{array}{l}
\chi_{1}(\xi) \\
\chi_{2}(\xi)
\end{array}\right)= & \left(\begin{array}{l}
\kappa_{1}\left[\Lambda_{1}+o(1)\right] e^{\left(\left(c-\sqrt{c^{2}+4 \mu_{1}}\right) / 2\right) \xi} \\
\kappa_{1}\left[\Gamma_{1}+o(1)\right] e^{\left(\left(c-\sqrt{c^{2}+4 \mu_{1}}\right) / 2\right) \xi}
\end{array}\right) \\
& +\left(\begin{array}{c}
\kappa_{2}\left[\Lambda_{2}+o(1)\right] e^{\left(\left(c-\sqrt{c^{2}+4 \mu_{2}}\right) / 2\right) \xi} \\
\kappa_{2}\left[\Gamma_{2}+o(1)\right] e^{\left(\left(c-\sqrt{c^{2}+4 \mu_{2}}\right) / 2\right) \xi}
\end{array}\right) .
\end{aligned}
$$


Furthermore, we can obtain that

$$
\begin{aligned}
\chi_{1}(\xi)= & \kappa_{1} \Lambda_{1} e^{\left(\left(c-\sqrt{c^{2}+4 \mu_{1}}\right) / 2\right) \xi}+\kappa_{2} \Lambda_{2} e^{\left(\left(c-\sqrt{c^{2}+4 \mu_{2}}\right) / 2\right) \xi} \\
& +\Omega_{11}(\xi)+\Omega_{12}(\xi), \\
\chi_{2}(\xi)= & \left.\kappa_{1} \Gamma_{1} e^{\left(\left(c-\sqrt{c^{2}+4 \mu_{1}}\right) / 2\right) \xi}+\kappa_{2} \Gamma_{2} e^{\left(\left(c-\sqrt{c^{2}+4 \mu_{2}}\right) / 2\right.}\right) \xi \\
& +\Omega_{21}(\xi)+\Omega_{22}(\xi),
\end{aligned}
$$

where

$$
\begin{array}{ll}
\lim _{\xi \rightarrow+\infty} \frac{\Omega_{11}(\xi)}{e^{\left(\left(c-\sqrt{c^{2}+4 \mu_{1}}\right) / 2\right) \xi}}=0, & \lim _{\xi \rightarrow+\infty} \frac{\Omega_{12}(\xi)}{e^{\left(\left(c-\sqrt{c^{2}+4 \mu_{2}}\right) / 2\right) \xi}}=0, \\
\lim _{\xi \rightarrow+\infty} \frac{\Omega_{21}(\xi)}{e^{\left(\left(c-\sqrt{c^{2}+4 \mu_{1}}\right) / 2\right) \xi}}=0, & \lim _{\xi \rightarrow+\infty} \frac{\Omega_{22}(\xi)}{e^{\left(\left(c-\sqrt{c^{2}+4 \mu_{2}}\right) / 2\right) \xi}}=0 .
\end{array}
$$

$\kappa_{1}, \kappa_{2}, \Lambda_{1}, \Lambda_{2}, \Gamma_{1}$, and $\Gamma_{2}$ are all constants.

Let $\mu=\min \left\{\mu_{1}, \mu_{2}\right\}$, then

$$
\begin{aligned}
& \lim _{\xi \rightarrow+\infty} \frac{\chi_{1}(\xi)}{e^{\left(\left(c-\sqrt{c^{2}+4 \mu}\right) / 2\right) \xi}} \\
& =\kappa_{1} \Lambda_{1} \lim _{\xi \rightarrow+\infty} e^{\left(\left(\sqrt{c^{2}+4 \mu}-\sqrt{c^{2}+4 \mu_{1}}\right) / 2\right) \xi} \\
& +\kappa_{2} \Lambda_{2} \lim _{\xi \rightarrow+\infty} e^{\left(\left(\sqrt{c^{2}+4 \mu}-\sqrt{c^{2}+4 \mu_{2}}\right) / 2\right) \xi} \\
& +\lim _{\xi \rightarrow+\infty} \frac{\Omega_{11}(\xi)}{e^{\left(\left(c-\sqrt{c^{2}+4 \mu_{1}}\right) / 2\right) \xi}} \lim _{\xi \rightarrow+\infty} e^{\left(\left(\sqrt{c^{2}+4 \mu}-\sqrt{c^{2}+4 \mu_{1}}\right) / 2\right) \xi} \\
& +\lim _{\xi \rightarrow+\infty} \frac{\Omega_{12}(\xi)}{e^{\left(\left(c-\sqrt{c^{2}+4 \mu_{2}}\right) / 2\right)} \xi \xi \rightarrow+\infty} \lim _{\left.\xi \rightarrow\left(\sqrt{c^{2}+4 \mu}-\sqrt{c^{2}+4 \mu_{2}}\right) / 2\right) \xi} \\
& =\Delta_{1}\left(\kappa_{1}, \kappa_{2}, \Lambda_{1}, \Lambda_{2}\right) \text {, } \\
& \lim _{\xi \rightarrow+\infty} \frac{\chi_{2}(\xi)}{e^{\left(\left(c-\sqrt{c^{2}+4 \mu}\right) / 2\right) \xi}} \\
& =\kappa_{1} \Gamma_{1} \lim _{\xi \rightarrow+\infty} e^{\left(\left(\sqrt{c^{2}+4 \mu}-\sqrt{c^{2}+4 \mu_{1}}\right) / 2\right) \xi} \\
& +\kappa_{2} \Gamma_{2} \lim _{\xi \rightarrow+\infty} e^{\left(\left(\sqrt{c^{2}+4 \mu}-\sqrt{c^{2}+4 \mu_{2}}\right) / 2\right) \xi} \\
& +\lim _{\xi \rightarrow+\infty} \frac{\Omega_{21}(\xi)}{e^{\left(\left(c-\sqrt{c^{2}+4 \mu_{1}}\right) / 2\right)} \xi} \lim _{\xi \rightarrow+\infty} e^{\left(\left(\sqrt{c^{2}+4 \mu}-\sqrt{c^{2}+4 \mu_{1}}\right) / 2\right) \xi} \\
& +\lim _{\xi \rightarrow+\infty} \frac{\Omega_{22}(\xi)}{e^{\left(\left(c-\sqrt{c^{2}+4 \mu_{2}}\right) / 2\right) \xi}} \lim _{\xi \rightarrow+\infty} e^{\left(\left(\sqrt{c^{2}+4 \mu}-\sqrt{c^{2}+4 \mu_{2}}\right) / 2\right) \xi} \\
& =\Delta_{2}\left(\kappa_{1}, \kappa_{2}, \Gamma_{1}, \Gamma_{2}\right) \text {, }
\end{aligned}
$$

where

$$
\begin{gathered}
\Delta_{1}\left(\kappa_{1}, \kappa_{2}, \Lambda_{1}, \Lambda_{2}\right)= \begin{cases}\kappa_{1} \Lambda_{1}, & \mu_{1}<\mu_{2}, \\
\kappa_{2} \Lambda_{2}, & \mu_{1}>\mu_{2},\end{cases} \\
\Delta_{2}\left(\kappa_{1}, \kappa_{2}, \Gamma_{1}, \Gamma_{2}\right)= \begin{cases}\kappa_{1} \Gamma_{1}, & \mu_{1}<\mu_{2}, \\
\kappa_{2} \Gamma_{2}, & \mu_{1}>\mu_{2} ;\end{cases}
\end{gathered}
$$

thus, when $\xi \rightarrow+\infty$, we can get that

$$
\begin{aligned}
& \left(\begin{array}{l}
\chi_{1}(\xi) \\
\chi_{2}(\xi)
\end{array}\right) \\
& \quad=\left(\begin{array}{c}
\Delta_{1}\left(\kappa_{1}, \kappa_{2}, \Lambda_{1}, \Lambda_{2}\right)(1+o(1)) e^{\left(\left(c-\sqrt{c^{2}+4 \mu}\right) / 2\right) \xi} \\
\Delta_{2}\left(\kappa_{1}, \kappa_{2}, \Gamma_{1}, \Gamma_{2}\right)(1+o(1)) e^{\left(\left(c-\sqrt{c^{2}+4 \mu}\right) / 2\right) \xi}
\end{array}\right) .
\end{aligned}
$$

In the case of (ii) $\mu_{1}=\mu_{2}$, we can obtain that the solution of model (77) has the following form:

$$
\begin{aligned}
\left(\psi_{1}, \widetilde{\psi}_{1}, \psi_{2}, \widetilde{\psi}_{2}\right)^{T}= & \left(G_{1}+G_{2} \xi\right) H_{1,2} e^{\overline{\lambda_{1}} \xi} \\
& +\left(G_{3}+G_{4} \xi\right) H_{3,4} e^{\overline{\lambda_{3}} \xi}
\end{aligned}
$$

where $H_{1,2}$ is the eigenvector of the constant matrix with $\overline{\lambda_{1}}=$ $\overline{\lambda_{2}}$ as the corresponding eigenvalues, $H_{3,4}$ is the eigenvector of the constant matrix with $\overline{\lambda_{3}}=\overline{\lambda_{4}}$ as the corresponding eigenvalues, $G_{i}(i=1,2,3,4)$ are arbitrary constants.

Since $\lim _{\xi \rightarrow+\infty}\left(\psi_{1}, \widetilde{\psi}_{1}, \psi_{2}, \widetilde{\psi}_{2}\right)^{T}=0$, thus

$$
\left(\psi_{1}, \widetilde{\psi}_{1}, \psi_{2}, \widetilde{\psi}_{2}\right)^{T}=\left(G_{3}+G_{4} \xi\right) H_{3,4} e^{\overline{\lambda_{3}} \xi} .
$$

So, when $\xi \rightarrow+\infty$, we can get that

$$
\left(\begin{array}{l}
\chi_{1}(\xi) \\
\chi_{2}(\xi)
\end{array}\right)=\left(\begin{array}{l}
\left(G_{1,3}+G_{1,4} \xi\right) e^{\overline{\lambda_{3}} \xi}+o\left(e^{\overline{\lambda_{3}} \xi}\right) \\
\left(G_{2,3}+G_{2,4} \xi\right) e^{\overline{\lambda_{4}} \xi}+o\left(e^{\overline{\lambda_{4} \xi}}\right)
\end{array}\right)
$$

By comparing the upper solution and roughness of the exponential dichotomy [24], we obtain the asymptotic decay rate of the traveling wave solutions at $+\infty$ given in Theorem 11 .

According to the monotone iteration process [3], the traveling wave solution $U(\xi)$ is increasing; thus $U^{\prime}(\xi) \geq 0$ and $U^{\prime}(\xi)=\left(\chi_{1}, \chi_{2}\right)^{T}(\xi)$ hold

$$
\begin{aligned}
& \chi_{1}^{\prime \prime}-c \chi_{1}^{\prime}+\frac{\partial F_{1}}{\partial S} \chi_{1}+\frac{\partial F_{1}}{\partial I} \chi_{2}=0 \\
& \chi_{2}^{\prime \prime}-c \chi_{2}^{\prime}+\frac{\partial F_{2}}{\partial S} \chi_{1}+\frac{\partial F_{2}}{\partial I} \chi_{2}=0
\end{aligned}
$$

satisfying

$$
\left(\chi_{1}, \chi_{2}\right)^{T}(\xi) \geq 0, \quad\left(\chi_{1}, \chi_{2}\right)^{T}( \pm \infty)=0 .
$$

The strong Maximum Principle implies that $\left(\chi_{1}, \chi_{2}\right)^{T}(\xi)$ $>0$. So the strict monotonicity of the traveling wave solutions is concluded. 
Now, we use the Sliding domain method to prove the uniqueness of the traveling wave solution. Let $U_{1}(\xi)=$ $\left(S_{1}, I_{1}\right)^{T}(\xi)$ and $U_{2}(\xi)=\left(S_{2}, I_{2}\right)^{T}(\xi)$ be the traveling wave solution of model (24), with $c>2 \sqrt{R_{0}-1}$. Thus, there are some positive numbers $A_{i}, B_{i}(i=1,2,3,4)$, such that for a big enough number $N \gg 1$, when $\xi<-N$, we have

$$
\begin{aligned}
\left(\begin{array}{l}
S_{1}(\xi) \\
I_{1}(\xi)
\end{array}\right)= & \left(\begin{array}{l}
A_{1} e^{\left(\left(c-\sqrt{c^{2}-4\left(R_{0}-1\right)}\right) / 2\right) \xi} \\
A_{2} e^{\left(\left(c-\sqrt{c^{2}-4\left(R_{0}-1\right)}\right) / 2\right) \xi}
\end{array}\right) \\
& +o\left(e^{\left(\left(c-\sqrt{c^{2}-4\left(R_{0}-1\right)}\right) / 2\right) \xi}\right) \\
\left(\begin{array}{c}
S_{2}(\xi) \\
I_{2}(\xi)
\end{array}\right)= & \left(\begin{array}{l}
A_{3} e^{\left(\left(c-\sqrt{c^{2}-4\left(R_{0}-1\right)}\right) / 2\right) \xi} \\
A_{4} e^{\left(\left(\left(c-\sqrt{c^{2}-4\left(R_{0}-1\right)}\right) / 2\right) \xi\right.}
\end{array}\right) \\
& +o\left(e^{\left(\left(c-\sqrt{c^{2}-4\left(R_{0}-1\right)}\right) / 2\right) \xi}\right)
\end{aligned}
$$

when $\xi>N$,

$$
\begin{aligned}
\left(\begin{array}{l}
S_{1}(\xi) \\
I_{1}(\xi)
\end{array}\right)= & \left(\begin{array}{l}
\widehat{S}^{*}-B_{1} e^{\left(\left(c-\sqrt{c^{2}+4 \mu}\right) / 2\right) \xi} \\
\widehat{I}^{*}-B_{2} e^{\left(\left(c-\sqrt{c^{2}+4 \mu}\right) / 2\right) \xi}
\end{array}\right) \\
& +o\left(e^{\left(\left(c-\sqrt{c^{2}+4 \mu}\right) / 2\right) \xi}\right), \\
\left(\begin{array}{c}
S_{2}(\xi) \\
I_{2}(\xi)
\end{array}\right)= & \left(\begin{array}{l}
\widehat{S}^{*}-B_{3} e^{\left(\left(c-\sqrt{c^{2}+4 \mu}\right) / 2\right) \xi} \\
\widehat{I}^{*}-B_{4} e^{\left(\left(c-\sqrt{c^{2}+4 \mu}\right) / 2\right) \xi}
\end{array}\right) \\
& +o\left(e^{\left(\left(c-\sqrt{c^{2}+4 \mu}\right) / 2\right) \xi}\right) .
\end{aligned}
$$

Since the traveling wave solutions of model (24) are translation-invariant, then for any $\theta \in R, U_{1}^{\theta}(\xi) \equiv U_{1}(\xi+\theta) \equiv$ $\left(S_{1}(\xi+\theta), I_{1}(\xi+\theta)\right)^{T}$ is also a traveling wave solution of model (24). Thus, by using the same method as above, when $\xi<-N$, we can get

$$
\begin{aligned}
& \left(\begin{array}{l}
S_{1}(\xi+\theta) \\
I_{1}(\xi+\theta)
\end{array}\right) \\
& =\left(\begin{array}{l}
A_{1} e^{\left(\left(c-\sqrt{c^{2}-4\left(R_{0}-1\right)}\right) / 2\right) \theta} e^{\left(\left(c-\sqrt{c^{2}-4\left(R_{0}-1\right)}\right) / 2\right) \xi} \\
A_{2} e^{\left(\left(c-\sqrt{c^{2}-4\left(R_{0}-1\right)}\right) / 2\right) \theta} e^{\left(\left(c-\sqrt{\left.c^{2}-4\left(R_{0}-1\right)\right)} / 2\right) \xi\right.}
\end{array}\right) \\
& \quad+o\left(e^{\left(\left(c-\sqrt{\left.c^{2}-4\left(R_{0}-1\right)\right)} / 2\right) \xi\right.}\right),
\end{aligned}
$$

when $\xi>N$,

$$
\begin{aligned}
& \left(\begin{array}{c}
S_{1}(\xi+\theta) \\
I_{1}(\xi+\theta)
\end{array}\right) \\
& =\left(\begin{array}{l}
\widehat{S}^{*}-B_{1} e^{\left(\left(c-\sqrt{c^{2}+4 \mu}\right) / 2\right) \theta} e^{\left(\left(c-\sqrt{c^{2}+4 \mu}\right) / 2\right) \xi} \\
\widehat{I}^{*}-B_{2} e^{\left(\left(c-\sqrt{c^{2}+4 \mu}\right) / 2\right) \theta} e^{\left(\left(c-\sqrt{c^{2}+4 \mu}\right) / 2\right) \xi}
\end{array}\right) \\
& \quad+o\left(e^{\left(\left(c-\sqrt{c^{2}+4 \mu}\right) / 2\right) \xi}\right) .
\end{aligned}
$$

If $\theta$ is large enough, then we can obtain the following inequalities:

$$
\begin{gathered}
A_{1} e^{\left(\left(c+\sqrt{c^{2}-4\left(R_{0}-1\right)}\right) / 2\right) \theta}>A_{3}, \\
A_{2} e^{\left(\left(c+\sqrt{c^{2}-4\left(R_{0}-1\right)}\right) / 2\right) \theta}>A_{4}, \\
B_{1} e^{\left(\left(c-\sqrt{c^{2}+4 \mu}\right) / 2\right) \theta}<B_{3}, \\
B_{2} e^{\left(\left(c-\sqrt{c^{2}+4 \mu}\right) / 2\right) \theta}<B_{4} .
\end{gathered}
$$

Thus, if $\theta$ is large enough, then $U_{1}^{\theta}(\xi)>U_{2}(\xi)$, for all $\xi \in$ $R \backslash[-N, N]$.

Now, we consider model $(24)$ on the interval $[-N, N]$.

First, suppose that

$$
W(\xi) \equiv U_{1}^{\theta}(\xi)-U_{2}(\xi) \geq 0, \quad \xi \in[-N, N],
$$

then

$$
\begin{aligned}
& W^{\prime \prime}-c W^{\prime} \\
& +\left(\begin{array}{ll}
\frac{\partial F_{1}}{\partial S}\left(S_{2}+\zeta_{1} \omega_{1}, I_{1}\right) & \frac{\partial F_{1}}{\partial I}\left(S_{1}, I_{2}+\zeta_{2} \omega_{2}\right) \\
\frac{\partial F_{2}}{\partial S}\left(S_{2}+\zeta_{3} \omega_{1}, I_{1}\right) & \frac{\partial F_{2}}{\partial I}\left(S_{1}, I_{2}+\zeta_{4} \omega_{2}\right)
\end{array}\right) W=0, \\
& W(-N)>0, \quad W(N)>0,
\end{aligned}
$$

where, $\zeta_{i} \in(0,1)(i=1,2,3,4), \xi \in(-N, N)$. Since the above model is monotone, by the Maximum Principle, we can deduce that $W(\xi)>0, \xi \in[-N, N]$. Consequently, we get that $U_{1}^{\theta}(\xi)>U_{2}(\xi), \xi \in R$.

Second, we suppose that there exists a point $\xi_{*} \in(-N, N)$ such that

$$
S_{1}^{\theta}\left(\xi_{*}\right)<S_{2}\left(\xi_{*}\right)
$$

or

$$
I_{1}^{\theta}\left(\xi_{*}\right)<I_{2}\left(\xi_{*}\right) .
$$

In this case, we increase $\theta$, that is shifting $U_{1}^{\theta}$ to the left, so that $U_{1}^{\theta}(-N)>U_{2}(-N)$ and $U_{1}^{\theta}(N)>U_{2}(N)$. According to the monotonicity of $U_{1}^{\theta}$ and $U_{2}$, we can find a number $\bar{\theta} \epsilon$ $(0,2 N)$ such that $U_{1}^{\theta}(\xi+\bar{\theta})>U_{2}(\xi), \xi \in(-N, N)$. Shifting $U_{1}^{\theta}(\xi+\bar{\theta})$ back until one component of $U_{1}^{\theta}(\xi+\bar{\theta})$ touches its counterpart of $U_{2}(\xi)$ at some point $\overline{\bar{\xi}} \in(-N, N)$. Since $U_{1}^{\theta}(\xi+$ $\bar{\theta})$ and $U_{2}(\xi)$ are strictly increasing, $\overline{\bar{\xi}} \in(-N, N)$, thus, we get that $U_{1}^{\theta}(\xi+\bar{\theta})>U_{2}(\xi), \xi= \pm N$. However, by the Maximum Principle for that component again, we find that components of $U_{1}^{\theta}$ and $U_{2}$ are identically equal for all $\xi \in[-N, N]$ for a larger number $\theta$. This is a contradiction, thus $U_{1}^{\theta}(\xi)>U_{2}(\xi)$, $\xi \in R$. Here, $\theta$ is a new number which is chosen by the above mean.

Now, decrease the $\theta$ until one of the following happens. 
Case (a). There is a $\bar{\theta} \geq 0$, such that $U_{1}^{\bar{\theta}}=U_{2}(\xi), \xi \in R$. In this case, we have finished the proof.

Case (b). There are a $\bar{\theta}$ and a point $\xi_{1} \in R$, such that one of the components of $U_{1}^{\bar{\theta}}$ and $U_{2}$ are equal. And $U_{1}^{\bar{\theta}} \geq U_{2}, \xi \in R$. On $R$ for that component, according to the Maximum Principle, we find that $U_{1}^{\bar{\theta}}$ and $U_{2}$ must be identical on that component. We can return to Case (a).

Consequently, in either situation, their exists a number $\bar{\theta} \geq 0$ such that

$$
U_{1}^{\bar{\theta}}(\xi)=U_{2}(\xi), \quad \xi \in(-\infty,+\infty) .
$$

This ends of the proof.

By Theorem 11, we can get the following theorem:

Theorem 12. For each $c \geq 2 \sqrt{R_{0}-1}$, model (3) has a unique (up to a translation of the origin) traveling wave solution. The traveling wave solution is strictly increasing and has the following asymptotic properties:

(i) $c>2 \sqrt{R_{0}-1}$ : when $\xi \rightarrow-\infty$,

$$
\begin{gathered}
\left.S(\xi)=\frac{R_{d}-1}{R_{d}}-A_{1} e^{\left(\left(c-\sqrt{c^{2}-4\left(R_{0}-1\right)}\right) / 2\right.}\right) \xi \\
+o\left(e^{\left(\left(c-\sqrt{c^{2}-4\left(R_{0}-1\right)}\right) / 2\right) \xi}\right), \\
I(\xi)=A_{2} e^{\left(\left(c-\sqrt{c^{2}-4\left(R_{0}-1\right)}\right) / 2\right) \xi}+o\left(e^{\left(\left(c-\sqrt{c^{2}-4\left(R_{0}-1\right)}\right) / 2\right) \xi}\right) .
\end{gathered}
$$

when $\xi \rightarrow+\infty$, and if $\mu_{1} \neq \mu_{2}$, then

$$
\begin{aligned}
S(\xi)= & S^{*}+\overline{A_{1}} e^{\left(\left(c-\sqrt{c^{2}+4 \mu}\right) / 2\right) \xi} \\
& +o\left(e^{\left(\left(c-\sqrt{c^{2}+4 \mu}\right) / 2\right) \xi}\right), \\
I(\xi)= & I^{*}-\overline{A_{2}} e^{\left(\left(c-\sqrt{c^{2}+4 \mu}\right) / 2\right) \xi} \\
& +o\left(e^{\left(\left(c-\sqrt{c^{2}+4 \mu}\right) / 2\right) \xi}\right),
\end{aligned}
$$

if $\mu_{1}=\mu_{2}$,

$$
\begin{aligned}
S(\xi)= & S^{*}+\left(\overline{\overline{A_{11}}}+\overline{\overline{A_{12}}} \xi\right) e^{\left(\left(c-\sqrt{c^{2}+4 \mu}\right) / 2\right) \xi} \\
& +o\left(e^{\left(\left(c-\sqrt{c^{2}+4 \mu}\right) / 2\right) \xi}\right) \\
I(\xi)= & I^{*}-\left(\overline{\overline{A_{21}}}+\overline{\overline{A_{22}}} \xi\right) e^{\left(\left(c-\sqrt{c^{2}+4 \mu}\right) / 2\right) \xi} \\
& +o\left(e^{\left(\left(c-\sqrt{c^{2}+4 \mu}\right) / 2\right) \xi}\right)
\end{aligned}
$$

where $\mu=\min \left\{\mu_{1}, \mu_{2}\right\}>0, \overline{\overline{A_{11}}}, \overline{\overline{A_{21}}} \in \mathbb{R} A_{1}, A_{2}, \overline{A_{1}}, \overline{A_{2}}$, $\overline{\overline{A_{12}}}$ and $\overline{\overline{A_{22}}}$ are all positive constants. (ii) $c=2 \sqrt{R_{0}-1}$ : when $\xi \rightarrow-\infty$,

$$
\begin{gathered}
S(\xi)=\frac{R_{d}-1}{R_{d}}-\left(A_{11}+A_{12} \xi\right) e^{\sqrt{R_{0}-1} \xi}+o\left(\xi e^{\sqrt{R_{0}-1} \xi}\right), \\
I(\xi)=\left(A_{11}+A_{12} \xi\right) e^{\sqrt{R_{0}-1} \xi}+o\left(\xi e^{\sqrt{R_{0}-1} \xi}\right) .
\end{gathered}
$$

when $\xi \rightarrow+\infty$, and if $\mu_{1} \neq \mu_{2}$, then

$$
\begin{aligned}
& S(\xi)=S^{*}+\overline{B_{11}} e^{\left(\sqrt{R_{0}-1}-\sqrt{R_{0}-1+\mu}\right) \xi}+o\left(e^{\left(\sqrt{R_{0}-1}-\sqrt{R_{0}-1+\mu}\right) \xi}\right), \\
& I(\xi)=I^{*}-\overline{B_{22}} e^{\left(\sqrt{R_{0}-1}-\sqrt{R_{0}-1+\mu}\right) \xi}+o\left(e^{\left(\sqrt{R_{0}-1}-\sqrt{R_{0}-1+\mu}\right) \xi}\right),
\end{aligned}
$$

if $\mu_{1}=\mu_{2}$, then

$$
\begin{aligned}
S(\xi)= & S^{*}+\left(\overline{\overline{B_{11}}}+\overline{\overline{B_{12}}} \xi\right) e^{\left(\sqrt{R_{0}-1}-\sqrt{R_{0}-1+\mu}\right) \xi} \\
& +o\left(e^{\left(\sqrt{R_{0}-1}-\sqrt{R_{0}-1+\mu}\right) \xi}\right), \\
I(\xi)= & I^{*}-\left(\overline{\overline{B_{21}}}+\overline{\overline{B_{22}}} \xi\right) e^{\left(\sqrt{R_{0}-1}-\sqrt{R_{0}-1+\mu}\right) \xi} \\
& +o\left(e^{\left(\sqrt{R_{0}-1}-\sqrt{R_{0}-1+\mu}\right) \xi}\right),
\end{aligned}
$$

where $\mu=\min \left\{\mu_{1}, \mu_{2}\right\}>0, B_{12}, B_{22}<0, B_{11}, B_{21}, \overline{\overline{B_{11}}}, \overline{\overline{B_{21}}} \epsilon$ $\mathbb{R}, \overline{B_{11}}, \overline{B_{22}}, \overline{\overline{B_{12}}}, \overline{\overline{B_{22}}}$ are all positive constants.

Theorem 13. There is no monotone traveling wave solution of model (24) for any $0<c<2 \sqrt{R_{0}-1}$. In other words, there is no monotone traveling wave solution of model (3) for any $0<c<2 \sqrt{R_{0}-1}$.

Proof. Suppose there is a monotone traveling wave solution $\llbracket(\xi)=\left(l_{1}(\xi), l_{2}(\xi)\right)^{T}$ of model $(24)$ with the wave speed $c_{0}$, where $c_{0} \in\left(0,2 \sqrt{R_{0}-1}\right)$.

The asymptotic model of $\mathbb{L}(\xi)=\left(l_{1}(\xi), l_{2}(\xi)\right)^{T}$ as $\xi \rightarrow$ $-\infty$ is

$$
\begin{gathered}
\bar{\lambda}^{\prime \prime}-c_{0} \bar{\lambda}^{\prime}-\nu\left(R_{d}-1\right) \bar{\lambda}+\left(\nu R_{d}+R_{0}-2 \nu\right) \bar{\mu}=0 \\
\bar{\mu}^{\prime \prime}-c_{0} \bar{\mu}^{\prime}+\left(R_{0}-1\right) \bar{\mu}=0
\end{gathered}
$$

The second function of (108) has two characteristics as the following ones: $\left(c_{0}+\sqrt{4\left(R_{0}-1\right)-c_{0}^{2}} i\right) / 2,\left(c_{0}-\right.$ $\left.\sqrt{4\left(R_{0}-1\right)-c_{0}^{2}} i\right) / 2$. Thus it has two independent solutions of the following form:

$$
\begin{aligned}
& \overline{\mu_{11}}=e^{\left(c_{0} / 2\right) \xi} \cos \left(\frac{\sqrt{4\left(R_{0}-1\right)-c_{0}^{2}} \xi}{2} \xi,\right. \\
& \overline{\mu_{22}}=e^{\left(c_{0} / 2\right) \xi} \sin \left(\frac{\sqrt{4\left(R_{0}-1\right)-c_{0}^{2}}}{2} \xi\right) .
\end{aligned}
$$


Similar to the proof of Theorem 11, we can get that, when $\xi \rightarrow-\infty, \chi_{2}(\xi)$ can be described as the following equation:

$$
\begin{aligned}
\chi_{2}(\xi)= & K_{1} e^{\left(c_{0} / 2\right) \xi} \cos \left(\frac{\left.\sqrt{4\left(R_{0}-1\right)-c_{0}^{2}} \xi\right)}{2} \xi\right)+\text { h.o.t, } \\
& +K_{2} e^{\left(c_{0} / 2\right) \xi} \sin \left(\frac{\sqrt{4\left(R_{0}-1\right)-c_{0}^{2}}}{2} \xi\right) \\
= & \sqrt{K_{1}^{2}+K_{2}^{2}} e^{\left(c_{0} / 2\right) \xi} \sin \left(\frac{\sqrt{4\left(R_{0}-1\right)-c_{0}^{2}}}{2}+\tau(\xi)\right) \\
& + \text { h.o.t, }
\end{aligned}
$$

where $\tan (\tau(\xi))=K_{1} / K_{2}$, and h.o.t is the short notation for the higher order terms.

That is to say, $l_{2}(\xi)$ is oscillating. Thus, any solution of model (24) with $0<c<2 \sqrt{R_{0}-1}$ is not strictly monotone.

Theorems 12 and 13 indicate that $c=2 \sqrt{R_{0}-1}$ is the critical minimal wave speed.

\section{Acknowledgments}

The authors would like to thank the anonymous referee for the very helpful suggestions and comments which led to improvements of our original paper. This research was supported by the Natural Science Foundation of Zhejiang Province (LY12A01014, R1110261, and LQ12A01009), the National Science Foundation of China (61272018), and the National Basic Research Program of China (2012CB426510).

\section{References}

[1] D. H. Sattinger, "Weighted norms for the stability of traveling waves," Journal of Differential Equations, vol. 25, no. 1, pp. 130$144,1977$.

[2] Y. Hosono and B. Ilyas, "Traveling waves for a simple diffusive epidemic model," Mathematical Models \& Methods in Applied Sciences, vol. 5, no. 7, pp. 935-966, 1995.

[3] J. Wu and X. Zou, "Traveling wave fronts of reaction-diffusion systems with delay," Journal of Dynamics and Differential Equations, vol. 13, no. 3, pp. 651-687, 2001.

[4] P. Weng and X. Q. Zhao, "Spreading speed and traveling waves for a multi-type SIS epidemic model," Journal of Differential Equations, vol. 229, no. 1, pp. 270-296, 2006.

[5] K. F. Zhang and X. Q. Zhao, "Spreading speed and travelling waves for a spatially discrete SIS epidemic model," Nonlinearity, vol. 21, no. 1, pp. 97-112, 2008.

[6] W. T. Li, G. Lin, and S. Ruan, "Existence of travelling wave solutions in delayed reaction-diffusion systems with applications to diffusion-competition systems," Nonlinearity, vol. 19, no. 6, pp. 1253-1273, 2006.
[7] S. Ma, "Traveling waves for non-local delayed diffusion equations via auxiliary equations," Journal of Differential Equations, vol. 237, no. 2, pp. 259-277, 2007.

[8] R. Liu, V. R. S. K. Duvvuri, and J. Wu, "Spread pattern formation of H5N1-avian influenza and its implications for control strategies," Mathematical Modelling of Natural Phenomena, vol. 3, no. 7, pp. 161-179, 2008.

[9] K. Li and X. Li, "Travelling wave solutions in diffusive and competition-cooperation systems with delays," IMA Journal of Applied Mathematics, vol. 74, no. 4, pp. 604-621, 2009.

[10] Z. C. Wang, W. T. Li, and S. Ruan, "Existence and stability of traveling wave fronts in reaction advection diffusion equations with nonlocal delay," Journal of Differential Equations, vol. 238, no. 1, pp. 153-200, 2007.

[11] Z. C. Wang and J. Wu, "Travelling waves of a diffusive KermackMcKendrick epidemic model with non-local delayed transmission," Proceedings of the Royal Society A, vol. 466, no. 2113, pp. 237-261, 2010.

[12] Z. C. Wang, J. Wu, and R. Liu, “Traveling waves of the spread of avian influenza," Proceedings of the American Mathematical Society, vol. 140, no. 11, pp. 3931-3946, 2012.

[13] A. Boumenir and V. M. Nguyen, "Perron theorem in the monotone iteration method for traveling waves in delayed reactiondiffusion equations," Journal of Differential Equations, vol. 244, no. 7, pp. 1551-1570, 2008.

[14] X. Hou and A. W. Leung, "Traveling wave solutions for a competitive reaction-diffusion system and their asymptotics," Nonlinear Analysis: Real World Applications, vol. 9, no. 5, pp. 21962213, 2008.

[15] X. Hou and W. Feng, "Traveling waves and their stability in a coupled reaction diffusion system," Communications on Pure and Applied Analysis, vol. 10, no. 1, pp. 141-160, 2011.

[16] Q. Gan, R. Xu, and P. Yang, "Travelling waves of a delayed SIRS epidemic model with spatial diffusion," Nonlinear Analysis: Real World Applications, vol. 12, no. 1, pp. 52-68, 2011.

[17] Q. Ye, Z. Li, M. Wang, and Y. Wu, An Introduction to ReactionDiffusion Equation, Science Press, Beijing, China, 2nd edition, 2011.

[18] H. W. Hethcote, "The mathematics of infectious diseases," SIAM Review, vol. 42, no. 4, pp. 599-653, 2000.

[19] Z. Ma, Y. Zhou, and J. Wu, Modeling and Dynamics of Infectious Diseases, vol. 11 of Series in Contemporary Applied Mathematics CAM, Higher Education Press, Beijing, China, 2009.

[20] W. Wang, Y. Cai, M. Wu, K. Wang, and Z. Li, "Complex dynamics of a reaction-diffusion epidemic model," Nonlinear Analysis: Real World Applications, vol. 13, no. 5, pp. 2240-2258, 2012.

[21] Y. Cai, W. Liu, Y. Wang, and W. Wang, "Complex dynamics of a diffusive epidemic model with strong Allee effect," Nonlinear Analysis: Real World Applications, vol. 14, no. 4, pp. 1907-1920, 2013.

[22] F. Berezovsky, G. Karev, B. Song, and C. Castillo-Chavez, "A simple epidemic model with surprising dynamics," Mathematical Biosciences and Engineering, vol. 2, no. 1, pp. 133-152, 2005.

[23] W. O. Kermack and A. G. McKendrick, "A contribution to the mathematical theory of epidemics," Proceedings of the Royal Society A, vol. 115, no. 772, pp. 700-721, 1927.

[24] E. A. Coddington and N. Levinson, Theory of Ordinary Differential Equations, Tata McGraw-Hill, New York, NY, USA, 1972. 


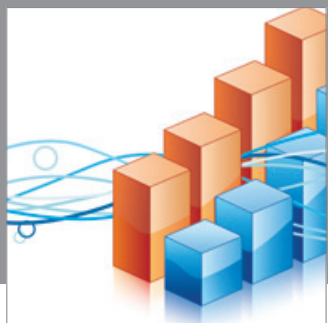

Advances in

Operations Research

mansans

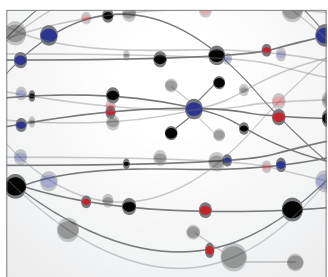

The Scientific World Journal
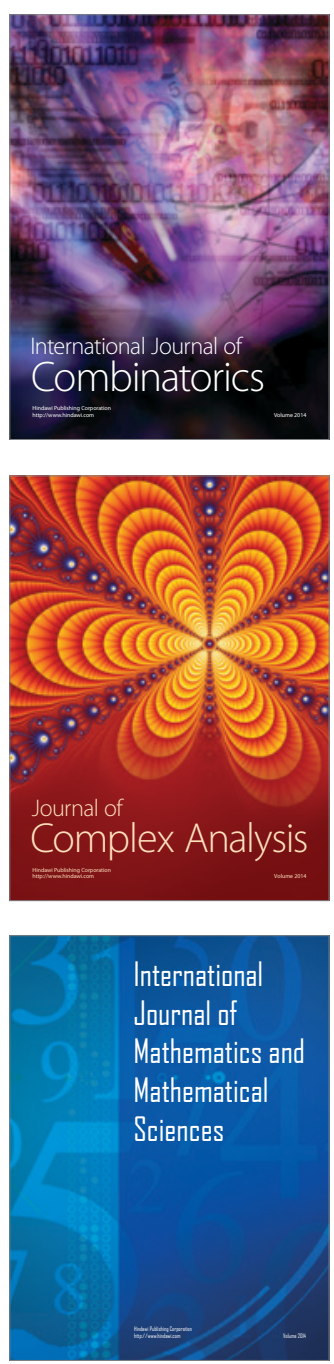
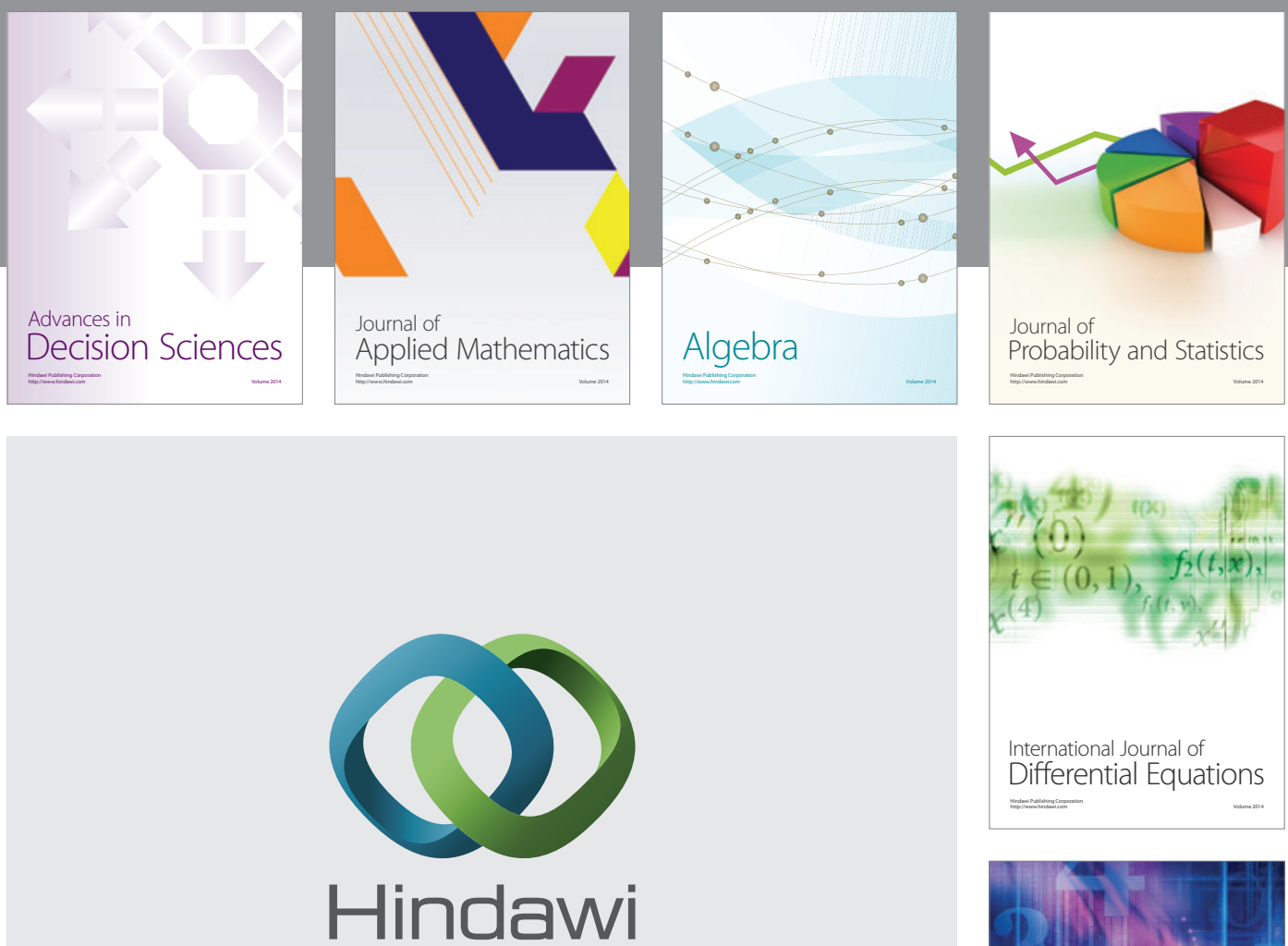

Submit your manuscripts at http://www.hindawi.com
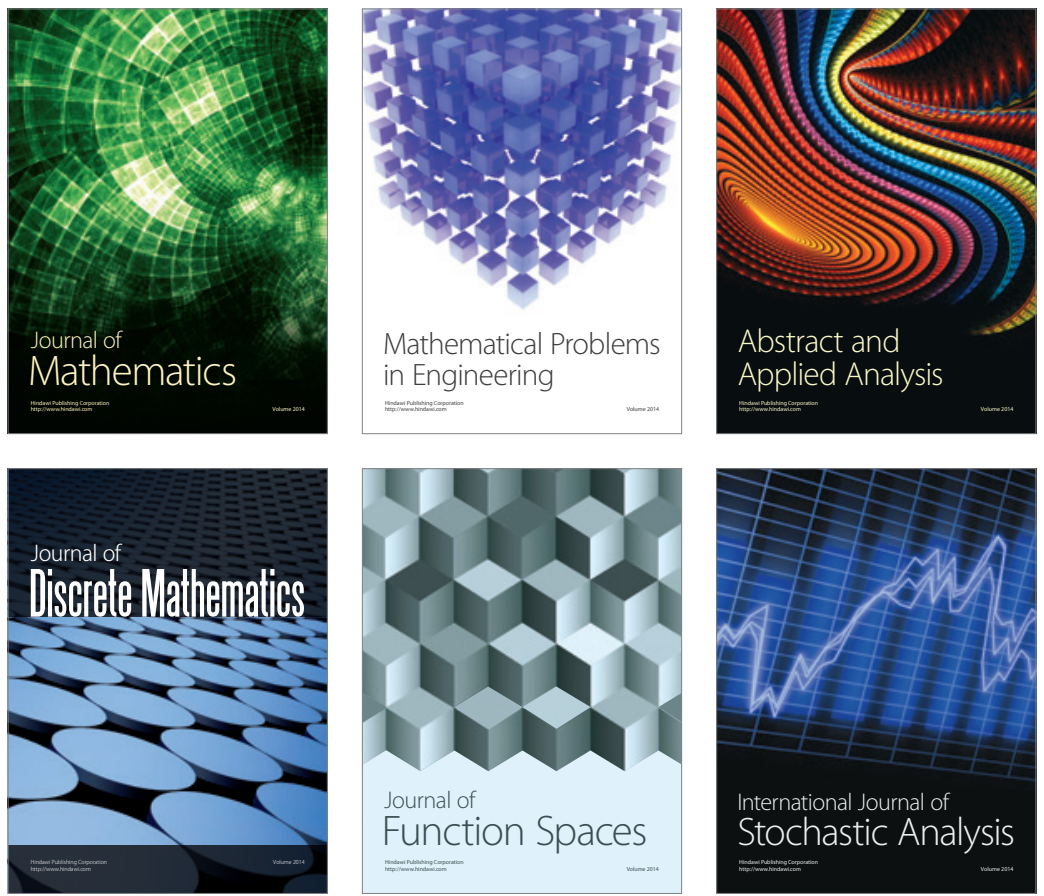

Journal of

Function Spaces

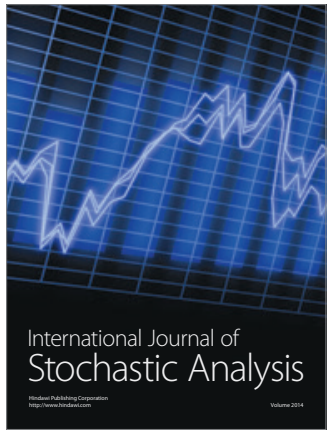

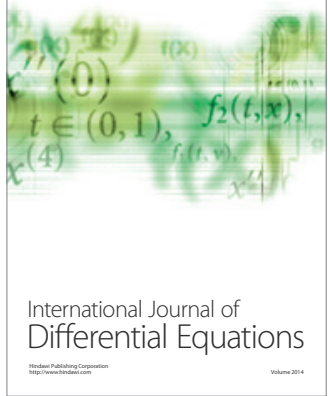
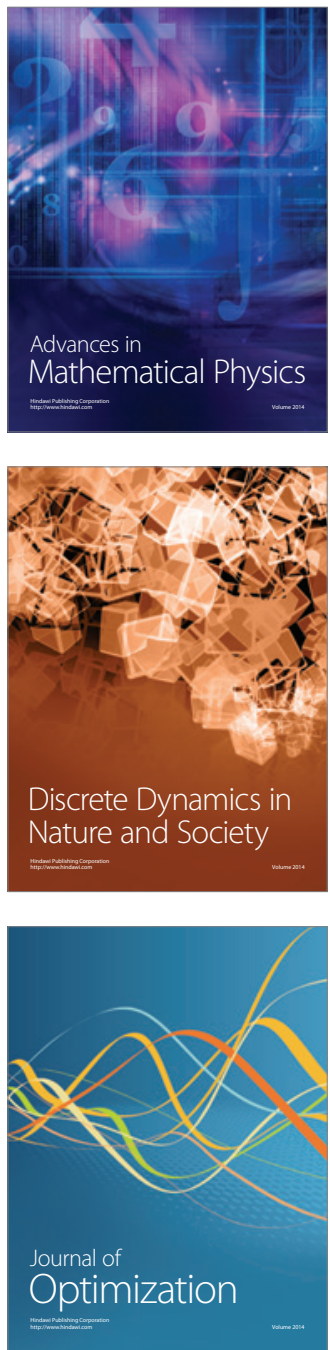